\title{
Linear hydrodynamics for driven granular gases
}

\author{
María Isabel García de Soria and Pablo Maynar \\ Física Teórica, Universidad de Sevilla, Apartado de Correos 1065, E-41080, Sevilla, Spain
}

\author{
Emmanuel Trizac \\ Laboratoire de Physique Théorique et Modèles Statistiques, UMR CNRS 8626, Université Paris-Sud, 91405 Orsay, France
}

(Received 27 November 2012; published 5 February 2013)

\begin{abstract}
We study the dynamics of a granular gas heated by a stochastic thermostat. From a Boltzmann description, we derive the hydrodynamic equations for small perturbations around the stationary state that is reached in the long time limit. Transport coefficients are identified as Green-Kubo formulas obtaining explicit expressions as a function of the inelasticity and the spatial dimension.
\end{abstract}

DOI: 10.1103/PhysRevE.87.022201

PACS number(s): 45.70.-n, 05.20.Dd, 05.60.-k, 51.10.+y

\section{INTRODUCTION}

Granular assemblies-composed of macroscopic solid bodies undergoing dissipative interactions-have been shown $[1,2]$ to frequently exhibit flows similar to those of normal fluids, and, for practical purposes, are often described by phenomenological hydrodynamic equations, i.e., equations for the density, flow velocity, and energy density. This is so even if energy is not a conserved variable (the condition is that the energy mode be a slow variable compared to the rest of excitations). When the dynamics of the grains can be partitioned into sequences of two-body collisions, there is support both from experiments and computer simulations for the usefulness of a kinetic theory description. This occurs, schematically, when there are no clusters nor jamming and the system remains fluidized [3]. In the low-density limit, the relevant dynamics is encoded in the one-particle distribution function, which obeys the inelastic Boltzmann equation [4,5]. This is the starting point for many of the formal derivations of hydrodynamic equations by applying similar tools and ideas as those used in the context of ordinary fluids [6]. In the free-cooling case the study of the existence and applicability of a hydrodynamic regime is rather complete for the inelastic hard sphere (IHS) model. The Navier-Stokes equations have been derived by the Chapman-Enskog expansion [7] and also via the linearized Boltzmann equation [8-10], yielding equivalent Green-Kubo formulas for the transport coefficients [11]. The problem for arbitrary densities has also been tackled in Refs. [12-14] applying linear response methods. Although the successful of the Navier-Stokes equations is remarkable, granular systems often require to go beyond this level of description. For these cases and close to a stationary state, a modification of the Chapman-Enskog expansion has been carried out, taking into account rheological effects [15,16].

The IHS model is also useful to rationalize driven situations, beyond the free-cooling case, where a stationary state is reached, see e.g., Refs. [17,18]. When in a vibrated system the stationary state is quasihomogeneous, or when the grains are immersed in an interstitial medium that acts as a thermostat [19-22], the setup can be effectively modelled as an IHS system driven by some random energy source. The energy injection can be performed by applying a random force to each particle. Depending on the stochastic properties of this force, different kinds of thermostats are obtained [23], one of the most used being the so-called stochastic thermostat, which consists of a white noise force acting on each grain [24-37]. This model has been less studied that its unforced version (freecooling case). To our knowledge, the only derivations of the hydrodynamic equations are the one made in Ref. [29] which consider some variants of the present stochastic thermostat in which the heating may depend on the local temperature. The objective of this work is to go further in this direction and to derive the hydrodynamic equations for the actual homogeneous stochastic thermostat model in the low-density limit. It is arguably the most commonly employed model in the simulations. At the Boltzmann equation level, we will consider states that are close to the homogeneous stationary regime, thereby obtaining linear equations for the deviations of the hydrodynamic fields around their homogeneous counterparts, with explicit Green-Kubo formulas for the transport coefficients. Let us note that, very recently, a study along the same lines has been performed in Ref. [37] by use of a different method, applying the Chapman-Enskog expansion to the Enskog equation.

The plan of the paper is as follows. The model is first defined in Sec. II. We summarize the main properties of the most general hydrodynamic state through which the stationary regime is reached in the long time limit. This state was analyzed in detail in Ref. [38] and, as will be shown, plays an essential role in the hydrodynamic description for the present model. In Sec. III, the linearized Boltzmann equation is written and the relevant modes for the hydrodynamic description are identified. These properties are subsequently exploited in Sec. IV to derive the linearized hydrodynamic equations. Finally, in Sec. V, we present a short summary of the results obtained in the paper while details of the calculations are given in seven appendixes, at the end of the text.

Before starting the analysis, a comment on the comparative merits of the Chapman-Enskog expansion and linearized Boltzmann approach is in order. The Chapman-Enskog method considers nonlinear excitations but assumes the existence of a normal solution. Within the linearized Boltzmann equation framework, linear excitations only are addressed, and scale separation is assumed. In this context, the asymptotic behaviors of the hydrodynamic fields are calculated as an exact property of the spectrum of the linearized Boltzmann operator. In addition, the analysis is somewhat simpler within the linear 
approach. In any case, it should be kept in mind that the Navier-Stokes equations must be the same independently of the method of derivation.

\section{THE MODEL}

Let us consider a dilute gas of $N$ smooth inelastic hard spheres $(d=3)$ or disks $(d=2)$ of mass $m$ and diameter $\sigma$. These bodies collide inelastically with a coefficient of normal restitution $\alpha$, independent of the relative velocity. If at time $t$ there is a binary encounter between particles $i$ and $j$, with velocities $\mathbf{V}_{i}(t)$ and $\mathbf{V}_{j}(t)$, respectively, the postcollisional velocities $\mathbf{V}_{i}^{\prime}(t)$ and $\mathbf{V}_{j}^{\prime}(t)$ are

$$
\begin{aligned}
\mathbf{V}_{i}^{\prime} & =\mathbf{V}_{i}-\frac{1+\alpha}{2}\left(\hat{\boldsymbol{\sigma}} \cdot \mathbf{V}_{i j}\right) \hat{\boldsymbol{\sigma}}, \\
\mathbf{V}_{j}^{\prime} & =\mathbf{V}_{j}+\frac{1+\alpha}{2}\left(\hat{\boldsymbol{\sigma}} \cdot \mathbf{V}_{i j}\right) \hat{\boldsymbol{\sigma}},
\end{aligned}
$$

where $\mathbf{V}_{i j} \equiv \mathbf{V}_{i}-\mathbf{V}_{j}$ is the relative velocity and $\hat{\boldsymbol{\sigma}}$ is the unit vector pointing from the center of particle $j$ to the center of particle $i$ at contact. Between collisions, the system is heated uniformly by adding a random velocity to the velocity of each particle independently with certain frequency and with a given probability distribution. Let us define the jump distribution, $P_{\Delta t}(\Delta \mathbf{v})$, as the probability that a particle experiences a jump $\Delta \mathbf{v}$ in the time interval $\Delta t$, that will be assumed to fulfill

$$
\begin{aligned}
\lim _{\Delta t \rightarrow 0} \int d \mathbf{y} y_{j} P_{\Delta t}(\mathbf{y}) & =0, \quad \lim _{\Delta t \rightarrow 0} \frac{1}{\Delta t} \int d \mathbf{y} y_{j}^{2} P_{\Delta t}(\mathbf{y})=\xi_{0}^{2} \\
j & =1, \ldots, d
\end{aligned}
$$

where $\xi_{0}^{2}$ is the strength of the noise. If the variance of this distribution is small compared to the velocity scale in which the one-particle distribution, $f(\mathbf{r}, \mathbf{v}, t)$, varies, the evolution equation is, in the low-density limit, the Boltzmann-FokkerPlanck equation [25,39],

$$
\begin{aligned}
& {\left[\frac{\partial}{\partial t}+\mathbf{v}_{1} \cdot \frac{\partial}{\partial \mathbf{r}_{1}}\right] f\left(x_{1}, t\right)} \\
& =\sigma^{d-1} \int d x_{2} \delta\left(\mathbf{r}_{12}\right) \bar{T}_{0}\left(\mathbf{v}_{1}, \mathbf{v}_{2}\right) f\left(x_{1}, t\right) f\left(x_{2}, t\right) \\
& \quad+\frac{\xi_{0}^{2}}{2} \frac{\partial^{2}}{\partial \mathbf{v}_{1}^{2}} f\left(x_{1}, t\right)
\end{aligned}
$$

where we have introduced the field variable $x \equiv\{\mathbf{r}, \mathbf{v}\}$ and the binary collision operator $\bar{T}_{0}$,

$$
\bar{T}_{0}\left(\mathbf{v}_{1}, \mathbf{v}_{2}\right)=\int d \hat{\boldsymbol{\sigma}} \Theta\left(\mathbf{v}_{12} \cdot \hat{\boldsymbol{\sigma}}\right)\left(\mathbf{v}_{12} \cdot \hat{\boldsymbol{\sigma}}\right)\left(\alpha^{-2} b_{\sigma}^{-1}-1\right) .
$$

Here the operator $b_{\sigma}^{-1}$ replaces the velocities $\mathbf{v}_{1}$ and $\mathbf{v}_{2}$ by the precollisional ones $\mathbf{v}_{1}^{*}$ and $\mathbf{v}_{2}^{*}$ given by

$$
\begin{aligned}
& \mathbf{v}_{1}^{*}=\mathbf{v}_{1}-\frac{1+\alpha}{2 \alpha}\left(\hat{\boldsymbol{\sigma}} \cdot \mathbf{v}_{12}\right) \hat{\boldsymbol{\sigma}}, \\
& \mathbf{v}_{2}^{*}=\mathbf{v}_{2}+\frac{1+\alpha}{2 \alpha}\left(\hat{\boldsymbol{\sigma}} \cdot \mathbf{v}_{12}\right) \hat{\boldsymbol{\sigma}} .
\end{aligned}
$$

Under the conditions noted above, the evolution equation does not depend on the details of the distribution $P_{\Delta t}$ but only on its second moment through the coefficient $\xi_{0}^{2}$.
It is convenient to introduce the hydrodynamic fields in the standard kinetic theory fashion, as the first velocity moments of the one-particle distribution function

$$
\begin{gathered}
n(\mathbf{r}, t)=\int d \mathbf{v} f(\mathbf{r}, \mathbf{v}, t), \\
n(\mathbf{r}, t) \mathbf{u}(\mathbf{r}, t)=\int d \mathbf{v} \mathbf{v} f(\mathbf{r}, \mathbf{v}, t), \\
\frac{d}{2} n(\mathbf{r}, t) T(\mathbf{r}, t)=\int d \mathbf{v} \frac{m}{2}[v-\mathbf{u}(\mathbf{r}, t)]^{2} f(\mathbf{r}, \mathbf{v}, t) .
\end{gathered}
$$

Let us also introduce the local thermal velocity through

$$
v(\mathbf{r}, t)=\left[\frac{2 T(\mathbf{r}, t)}{m}\right]^{1 / 2}
$$

We will see that, in terms of the homogeneous hydrodynamic fields, we can specify some relevant states at the Boltzmann equation level. It is known numerically that for a wide class of initial conditions, the system reaches a homogeneous stationary state [26]. Assuming that total momentum is zero, i.e., $\int d \mathbf{v v} f(\mathbf{v}, 0)=\mathbf{0}$, the state is characterized by an isotropic stationary distribution, $f_{s}(v)$, which was studied in detail in Ref. [25]. There, the distribution was written as

$$
f_{s}(v)=\frac{n}{v_{s}^{d}} \chi_{s}(c), \quad \mathbf{c}=\frac{\mathbf{v}}{v_{s}},
$$

where $n$ is the total density and $v_{s}$ is the stationary thermal velocity. We have also introduced the scaled distribution function, $\chi_{s}$, which is independent of the strength of the noise $\xi_{0}^{2}$, i.e., all the dependence of the distribution on $\xi_{0}^{2}$ is written in terms of the temperature. As this distribution is quite close to a Maxwellian, an expansion in terms of Sonine polynomials [6] does make sense. In the so-called first Sonine approximation the function reads [25]

$$
\chi_{s}(c) \approx \chi_{M}(c)\left[1+a_{2}^{s} S_{2}\left(c^{2}\right)\right]
$$

where $\chi_{M}$ is the Maxwellian distribution with unit temperature, $S_{2}\left(c^{2}\right)=\frac{d(d+2)}{8}-\frac{d+2}{2} c^{2}+\frac{1}{2} c^{4}$ is the second Sonine polynomial, and $a_{2}^{s}$ is the kurtosis of the distribution. Within this approximation and neglecting nonlinear contributions in $a_{2}^{s}$, the distribution function can be calculated as [25]

$$
a_{2}^{s}(\alpha)=\frac{16(1-\alpha)\left(1-2 \alpha^{2}\right)}{73+56 d-24 d \alpha-105 \alpha+30(1-\alpha) \alpha^{2}},
$$

with a stationary temperature,

$$
T_{s}=m\left[\frac{d \Gamma(d / 2) \xi_{0}^{2}}{2 \pi^{\frac{d-1}{2}}\left(1-\alpha^{2}\right) n \sigma^{d-1}}\left(1-\frac{3}{16} a_{2}^{s}\right)\right]^{2 / 3} .
$$

It has recently been shown that, in a homogeneous situation, the initial condition is "forgotten" before the stationary state has been reached [38]. The system approaches the stationary state through a universal route in which all the time dependence of the distribution function, $f_{H}(\mathbf{v}, t)$, goes through the instantaneous temperature, $T_{H}(t)$ (in the following we will denote the universal state with the subindex $H$ ). In contrast with the free-cooling case, due to the parameter $\xi_{0}^{2}$, we can construct an additional quantity with dimensions of temperature apart from the instantaneous temperature, namely the stationary 
temperature given by Eq. (13). Then, by dimensional analysis the distribution function has a two-parameter scaling form,

$$
f_{H}(\mathbf{v}, t)=\frac{n}{v_{H}(t)^{d}} \chi(c, \beta), \quad \mathbf{c}=\frac{\mathbf{v}}{v_{H}(t)}, \quad \beta=\frac{v_{s}}{v_{H}(t)} .
$$

Let us remark that we have redefined the variable compared to the one introduced in Eq. (10) and we have introduced the instantaneous thermal velocity, $v_{H}(t)$. In Ref. [38] it was shown that this state actually exists and the dynamics is partitioned in a first rapid stage where initial conditions matter and a subsequent universal relaxation towards stationarity, where only the distance to the steady state is relevant, through the dimensionless inverse typical velocity $\beta=v_{s} / v_{H}(t)$, i.e.,

$$
f\left(\mathbf{v}, t \mid f_{0}\right) \longrightarrow \frac{n}{v_{H}^{d}(t)} \chi(c, \beta) \longrightarrow f_{s}(v) .
$$

Let us note that a similar two parameter scaling occurs in the uniform shear flow of granular gases [40,41]. In this case, after a quick transient, the system forgets the initial condition and evolves to the stationary state through a normal state, where the role of $\beta$ is played by the dimensionless shear rate, $a^{*}=a\left[n \sigma^{d-1} v_{0}(t)\right]^{-1}$. As in the stationary state, numerical simulations show that the scaled distribution is close to a Maxwellian and it can be calculated in the first Sonine approximation,

$$
\chi(c, \beta) \approx \chi_{M}(c)\left[1+a_{2}(\beta) S_{2}\left(c^{2}\right)\right],
$$

where, by definition, we have

$$
\begin{gathered}
\int d \mathbf{c} \chi(c, \beta)=1, \\
\int d \mathbf{c c} \chi(c, \beta)=\mathbf{0}, \\
\int d \mathbf{c} c^{2} \chi(c, \beta)=\frac{d}{2} .
\end{gathered}
$$

Introducing the approximated distribution (16) into the Boltzmann equation, and neglecting the nonlinear terms in $a_{2}(\beta)$, it is possible to identify the universal distribution, i.e., the universal $a_{2}(\beta)$, to be characterized by [38]

$$
a_{2}(\beta)=a_{2}^{s}\left[1+\frac{1-\beta^{3}}{B-1}{ }_{2} F_{1}\left(-\frac{1}{3}, 1 ; \frac{4 B-1}{3} ; \beta^{3}\right)\right],
$$

valid for $0<\beta<1$, and

$a_{2}(\beta)=-\frac{4 B a_{2}^{s}}{7 \beta^{3}\left(1-1 / \beta^{3}\right)^{\frac{4 B}{3}}} 2 F_{1}\left(\frac{7}{3}, 1+\frac{4 B}{3} ; \frac{10}{3} ; \frac{1}{\beta^{3}}\right)$

for $\beta>1$. Here we have introduced the hypergeometric function, ${ }_{2} F_{1}$ [42], and the coefficient

$$
B=\frac{73+8 d(7-3 \alpha)+15 \alpha[2 \alpha(1-\alpha)-7]}{16(1-\alpha)\left(3+2 d+2 \alpha^{2}\right)+a_{2}^{s}[85+d(30 \alpha-62)+3 \alpha(10 \alpha(1-\alpha)-39)]}
$$

As in the long time limit the distribution tends to the stationary state we have

$$
\lim _{\beta \rightarrow 1^{+}} a_{2}(\beta)=\lim _{\beta \rightarrow 1^{-}} a_{2}(\beta)=a_{2}^{s} .
$$

We also have that the first derivative is continuous,

$$
\lim _{\beta \rightarrow 1^{+}} \frac{d}{d \beta} a_{2}(\beta)=\lim _{\beta \rightarrow 1^{-}} \frac{d}{d \beta} a_{2}(\beta),
$$

an important property that will be needed in the following sections.

The evolution equation for the temperature (or equivalently for the thermal velocity, $v_{H}$ ) in the universal state can be calculated by inserting the scaling form (14) into the Boltzmann equation and taking the second velocity moment. When this is done, we obtain

$$
\frac{d v_{H}(t)}{d t}=\ell^{-1}\left[\mu(1) \beta^{3}-\mu(\beta)\right] v_{H}(t)^{2},
$$

where we have introduced the dimensionless coefficient

$$
\mu(\beta)=-\frac{1}{2 d} \int d \mathbf{c}_{1} \int d \mathbf{c}_{2} \chi\left(c_{1}, \beta\right) \chi\left(c_{2}, \beta\right) T_{0}\left(\mathbf{c}_{1}, \mathbf{c}_{2}\right)\left(c_{1}^{2}+c_{2}^{2}\right) .
$$

The operator $T_{0}$ is

$$
T_{0}\left(\mathbf{c}_{1}, \mathbf{c}_{2}\right)=\int d \hat{\boldsymbol{\sigma}} \Theta\left(\mathbf{c}_{12} \cdot \hat{\boldsymbol{\sigma}}\right)\left(\mathbf{c}_{12} \cdot \hat{\boldsymbol{\sigma}}\right)\left(b_{\sigma}-1\right),
$$

where $b_{\sigma}$ replaces the velocities $\mathbf{v}_{1}$ and $\mathbf{v}_{2}$ by the postcollisional ones $\mathbf{v}_{1}^{\prime}$ and $\mathbf{v}_{2}^{\prime}$ given by

$$
\begin{aligned}
& \mathbf{v}_{1}^{\prime}=\mathbf{v}_{1}-\frac{1+\alpha}{2}\left(\hat{\boldsymbol{\sigma}} \cdot \mathbf{v}_{12}\right) \hat{\boldsymbol{\sigma}}, \\
& \mathbf{v}_{2}^{\prime}=\mathbf{v}_{2}+\frac{1+\alpha}{2}\left(\hat{\boldsymbol{\sigma}} \cdot \mathbf{v}_{12}\right) \hat{\boldsymbol{\sigma}} .
\end{aligned}
$$

By inserting the function $\chi$ in the first Sonine approximation, Eq. (16), an approximate evolution equation for $v_{H}$ can be obtained. Nevertheless, we will be only interested in situations where we are close to the stationary state, $\beta=1$, and then equation (23) can be linearized, obtaining

$$
\frac{d v_{H}(t)}{d t}=-\gamma \frac{v_{s}}{\ell}\left[v_{H}(t)-v_{s}\right],
$$

where we have introduced the dimensionless coefficient

$$
\gamma=3 \mu(1)-\left.\frac{d \mu(\beta)}{d \beta}\right|_{\beta=1} .
$$

The coefficient $\gamma$ is calculated in Appendix A in the first Sonine approximation. Let us note that the difference of Eq. (27) with the equivalent one in Ref. [26] is just the term $\left.\frac{d \mu(\beta)}{d \beta}\right|_{\beta=1}$, which, although small, is shown in the Appendix to be of the same order as $a_{2}$. The solution of Eq. (27) is

$$
v_{H}(t)=v_{s}+\left[v_{H}(0)-v_{s}\right] e^{-\gamma \frac{v_{s}}{\ell} t},
$$


which shows that the relaxation of $v_{H}$ to the stationary value is given in terms of the coefficient $\gamma$.

The universal state that we have identified, which in the following will be referred to as the $\beta$ state, is analogous, for heated granular gases, to the homogeneous cooling state for unforced systems [7]. It represents the most general homogeneous hydrodynamic state and, as will be seen in the next sections, its existence is crucial in the study of the relevance of a hydrodynamic description for granular gases heated by the stochastic thermostat.

\section{THE LINEARIZED BOLTZMANN EQUATION}

Let us consider states close to the stationarity, so we can write

$$
\delta f\left(x_{1}, t\right)=f\left(x_{1}, t\right)-f_{s}\left(v_{1}\right), \quad\left|\delta f\left(x_{1}, t\right)\right| \ll f_{s}\left(v_{1}\right) .
$$

The evolution equation for this function is obtained from the Boltzmann equation (3) by neglecting the nonlinear terms in $\delta f$, obtaining

$$
\frac{\partial}{\partial t} \delta f\left(x_{1}, t\right)+\mathbf{v}_{1} \cdot \frac{\partial}{\partial \mathbf{r}_{1}} \delta f\left(x_{1}, t\right)=K\left(\mathbf{v}_{1}\right) \delta f\left(x_{1}, t\right),
$$

where $K\left(\mathbf{v}_{1}\right)$ is a linear operator defined by

$$
K\left(\mathbf{v}_{1}\right)=\sigma^{d-1} \int d \mathbf{v}_{2} \bar{T}_{0}\left(\mathbf{v}_{1}, \mathbf{v}_{2}\right)\left(1+P_{12}\right) f_{s}\left(v_{2}\right)+\frac{\xi_{0}^{2}}{2} \frac{\partial^{2}}{\partial \mathbf{v}_{1}^{2}}
$$

with $P_{12}$ an operator that interchanges the label 1 and 2 in the function on which it acts. Now, let us introduce a dimensionless space variable as

$$
\mathbf{l}=\frac{\mathbf{r}}{\ell}, \quad \ell=\left(n \sigma^{d-1}\right)^{-1},
$$

where $\ell$ is proportional to the mean free path, and a dimensionless time,

$$
s=\frac{v_{s}}{\ell} t
$$

which is proportional to the number of collisions per particle in the interval $(0, t)$. It is also convenient to introduce the dimensionless distribution, $\delta \chi$, through

$$
\delta f(x, t)=\frac{n}{v_{s}^{d}} \delta \chi(\mathbf{l}, \mathbf{c}, s) .
$$

The equation for $\delta \chi$ reads

$$
\frac{\partial}{\partial s} \delta \chi(\mathbf{l}, \mathbf{c}, s)=\left[\Lambda(\mathbf{c})-\mathbf{c} \cdot \frac{\partial}{\partial \mathbf{l}}\right] \delta \chi(\mathbf{l}, \mathbf{c}, s) .
$$

Here we have introduced the homogeneous linearized Boltzmann collision operator, $\Lambda$, defined by

$$
\Lambda\left(\mathbf{c}_{1}\right)=\int d \mathbf{c}_{2} \bar{T}_{0}\left(\mathbf{c}_{1}, \mathbf{c}_{2}\right)\left(1+P_{12}\right) \chi_{s}\left(c_{2}\right)+\frac{\widetilde{\xi}^{2}}{2} \frac{\partial^{2}}{\partial \mathbf{c}_{1}^{2}},
$$

where

$$
\widetilde{\xi}^{2}=\frac{\xi_{0}^{2} \ell}{v_{s}^{3}} \approx \frac{\pi^{\frac{d-1}{2}}\left(1-\alpha^{2}\right)}{\sqrt{2} d \Gamma(d / 2)}\left(1+\frac{3}{16} a_{2}^{s}\right)
$$

is the dimensionless amplitude of the noise calculated in the first Sonine approximation. Since the equation is linear and the linearized Boltzmann collision operator does not change the space variable, it is convenient to introduce the Fourier component

$$
\delta \chi_{\mathbf{k}}(\mathbf{c}, s)=\int d \mathbf{l} e^{-i \mathbf{k} \cdot \mathbf{l}} \delta \chi(\mathbf{l}, \mathbf{c}, s) .
$$

For an infinite system or with periodic boundary conditions, the evolution equation for these components is

$$
\frac{\partial}{\partial s} \delta \chi_{\mathbf{k}}(\mathbf{c}, s)=[\Lambda(\mathbf{c})-i \mathbf{k} \cdot \mathbf{c}] \delta \chi_{\mathbf{k}}(\mathbf{c}, s)
$$

Equation (36) or the Fourier counterpart, Eq. (40), is the so-called linearized Boltzmann equation and it describes the dynamics of any small perturbation around the homogeneous stationary state. This provides us with our starting point for the study of the possibility of a hydrodynamic description close to the stationary state.

The solution of Eq. (40) can be written formally as

$$
\delta \chi_{\mathbf{k}}(\mathbf{c}, s)=e^{[\Lambda(\mathbf{c})-i \mathbf{k} \cdot \mathbf{c}] s} \delta \chi_{\mathbf{k}}(\mathbf{c}, 0),
$$

which shows clearly that the excitations of the gas are determined by the spectrum properties of the linear operator $\Lambda(\mathbf{c})-i \mathbf{k} \cdot \mathbf{c}$. This suggests the study of the eigenvalue problem

$$
[\Lambda(\mathbf{c})-i \mathbf{k} \cdot \mathbf{c}] \xi_{j}(\mathbf{k}, \mathbf{c})=\lambda_{j}(\mathbf{k}) \xi_{j}(\mathbf{k}, \mathbf{c}),
$$

which is posed in a Hilbert space of functions of $\mathbf{c}$ with scalar product

$$
\langle g \mid h\rangle=\int d \mathbf{c} \chi_{s}^{-1}(c) g^{*}(\mathbf{c}) h(\mathbf{c}),
$$

with $g^{*}$ the complex conjugate of $g$. The search for hydrodynamic excitations, which are defined as the ones associated to the slowest modes, can be carried out by assuming that the modes are analytic in $\mathbf{k}$ and looking first for the $\mathbf{k}=\mathbf{0}$ solution of Eq. (42). Let us then consider the homogeneous eigenvalue problem

$$
\Lambda(\mathbf{c}) \xi_{j}(\mathbf{c})=\lambda_{j} \xi_{j}(\mathbf{c})
$$

where we have introduced the notation $\xi_{j}(\mathbf{k}=\mathbf{0}, \mathbf{c})=\xi_{j}(\mathbf{c})$ and $\lambda_{j}(\mathbf{k}=\mathbf{0})=\lambda_{j}$. We will now see that the special universal solution studied in the previous section allows us to identify a family of exact solutions of the linearized Boltzmann equation related to $d+2$ modes of the homogeneous linearized collision operator, $\Lambda$. The idea is similar in spirit to the one introduced in Refs. [8-10] to calculate the hydrodynamic eigenfunctions in the free-cooling case. Let us consider the family of exact solutions of the homogeneous nonlinear Boltzmann equation

$$
f_{H}(\mathbf{v}, t)=\frac{\bar{n}}{\bar{v}_{H}(t)^{d}} \chi\left[\frac{\mathbf{v}-\mathbf{u}}{\bar{v}_{H}(t)}, \frac{\bar{v}_{s}}{\bar{v}_{H}(t)}\right],
$$

which are parameterized by the density, $\bar{n}$, the constant velocity flow, $\mathbf{u}$, and the thermal velocity, $\bar{v}_{H}(t)$. The bars on the quantities refer to variables that differ from $\left(n, v_{H}\right)$ introduced earlier, $\bar{v}_{H}(t)$ being the thermal velocity of the $\beta$ state corresponding to $\bar{n}$. If we consider states close to stationarity, the function $\bar{v}_{H}(t)$ is known and given by Eq. (29). But then the family

$$
\delta f(\mathbf{v}, t)=\frac{\bar{n}}{\bar{v}_{H}(t)^{d}} \chi\left[\frac{\mathbf{v}-\mathbf{u}}{\bar{v}_{H}(t)}, \frac{\bar{v}_{s}}{\bar{v}_{H}(t)}\right]-f_{s}(v),
$$


to linear order in the fields, has to be a solution of the linearized Boltzmann equation. In Appendix $\mathrm{B} \delta f$ is calculated to linear order, which leads to the corresponding scaled distribution,

$$
\begin{aligned}
\delta \chi(\mathbf{c}, s)= & \frac{\delta n}{n}\left[\chi_{s}(c)+\frac{1}{3} \frac{\partial}{\partial \mathbf{c}} \cdot\left[\mathbf{c} \chi_{s}(c)\right]\right]-\frac{\mathbf{u}}{v_{s}} \cdot \frac{\partial}{\partial \mathbf{c}} \chi_{s}(c) \\
& -\left[\frac{\delta n}{3 n}+\frac{\delta v_{H}(0)}{v_{s}}\right] e^{-\gamma s}\left[\frac{\partial}{\partial \mathbf{c}} \cdot\left[\mathbf{c} \chi_{s}(c)\right]\right. \\
& \left.+\left.\frac{\partial}{\partial \beta} \chi(c, \beta)\right|_{\beta=1}\right]
\end{aligned}
$$

where $\delta n=\bar{n}-n$ and $\delta v_{H}(0)=\bar{v}_{H}(0)-v_{s}$. The functions given by (47) generate a family of solutions of the homogeneous linearized Boltzmann equation, which can be seen as the superposition of $d+2$ modes. We, consequently, have identified the following eigenfunctions of $\Lambda$ :

$$
\begin{gathered}
\xi_{1}(c)=\chi_{s}(c)+\frac{1}{3} \frac{\partial}{\partial \mathbf{c}} \cdot\left[\mathbf{c} \chi_{s}(c)\right], \\
\xi_{2}(\mathbf{c})=-\frac{\partial}{\partial \mathbf{c}} \chi_{s}(c), \\
\xi_{3}(c)=-\frac{\partial}{\partial \mathbf{c}} \cdot\left[\mathbf{c} \chi_{s}(c)\right]-\left.\frac{\partial}{\partial \beta} \chi(c, \beta)\right|_{\beta=1},
\end{gathered}
$$

with the corresponding eigenvalues,

$$
\lambda_{1}=\lambda_{2}=0, \quad \lambda_{3}=-\gamma,
$$

where the null eigenvalue is $(d+1)$-fold degenerate. Moreover, Eq. (47) can be rewritten as

$$
\delta \chi(\mathbf{c}, s)=\sum_{j=1}^{3} e^{\lambda_{j} s}\left\langle\bar{\xi}_{j}(\mathbf{c}) \mid \delta \chi(\mathbf{c}, 0)\right\rangle \xi_{j}(\mathbf{c}),
$$

with the following definitions of the functions $\bar{\xi}_{i}$ :

$$
\bar{\xi}_{1}(\mathbf{c})=\chi_{s}(c), \quad \bar{\xi}_{2}(\mathbf{c})=\chi_{s}(c) \mathbf{c}, \quad \bar{\xi}_{3}=\chi_{s}(c)\left(\frac{c^{2}}{d}-\frac{1}{6}\right) .
$$

These functions are just the linear combinations of the the first two velocity moments, $\left\{1, \mathbf{c}, c^{2}\right\}$, normalized to enforce

$$
\left\langle\bar{\xi}_{i} \mid \xi_{j}\right\rangle=\delta_{i j}, \quad i, j=1,2,3 \text {. }
$$

In fact, the functions $\xi_{1}$ and $\boldsymbol{\xi}_{2}$ have been previously identified in Refs. [33,34,43], where they were used to study the fluctuations of quantities like the total energy in the stationary state. There, it was also proven that $\bar{\xi}_{1}$ and $\bar{\xi}_{2}$ are eigenfunctions of the adjoint operator of the linearized collision operator, $\Lambda^{+}$, associated to the null eigenvalue. This follows from the conservation of the number of particles and momentum:

$$
\int d \mathbf{c} \Lambda(\mathbf{c}) h(\mathbf{c})=\int d \mathbf{c} c_{i} \Lambda(\mathbf{c}) h(\mathbf{c})=0 .
$$

Then, if we assume that eigenfunctions of $\Lambda,\left\{\xi_{i}\right\}_{i=1}^{\infty}$, form a complete set, in the expansion

$$
f(\mathbf{c})=\sum_{j=1}^{\infty} C_{j} \xi_{j}(\mathbf{c})
$$

we have $C_{1}=\left\langle\bar{\xi}_{1} \mid f\right\rangle$ and $\mathbf{C}_{2}=\left\langle\overline{\boldsymbol{\xi}}_{2} \mid f\right\rangle$. With the aid of Eq. (47), we are able to identify a new eigenfunction, $\xi_{3}$, that, as seen in Eq. (50), depends on the derivative with respect to $\beta$ of the universal state $\chi$ in the stationary regime. Let us remark that $\bar{\xi}_{3}$ is not an eigenfunction of $\Lambda^{+}$. We only have $C_{3}=\left\langle\bar{\xi}_{3} \mid f\right\rangle$ if the function $f$ belongs to the space generated by the set $\left\{\xi_{i}\right\}_{i=1}^{3}$.

In the elastic case, i.e., $\alpha=1$ and $\xi_{0}^{2}=0$, the spectrum of $\Lambda(\mathbf{k}, \mathbf{c})$ has been analyzed in detail and it is known that the modes associated to the locally conserved quantities, the hydrodynamic modes, are the slowest ones in the $k \rightarrow 0$ limit, where the respective eigenvalues vanish [44]. Furthermore, it is known that the spectrum is analytic in $k$ around $k=0$ and that there is scale separation, i.e., the hydrodynamic modes are isolated from the rest of modes. For the inelastic linearized collision operator, no such result is available. We have nevertheless shown that $d+1$ of the $d+2$ identified modes are associated to the vanishing eigenvalue, and the remaining mode is associated to $\gamma$, which itself vanishes in the elastic limit. We therefore expect that a similar property will hold with the identified modes - at least close to the elastic limit, although we expect it to apply also beyond-and these modes will henceforth be coined hydrodynamic. In the following, we will assume that they are the slowest ones and that they are analytic. Under this proviso, the asymptotic behavior of the one particle distribution function for $k \ll 1, s \gg 1$ is

$$
\delta \chi_{\mathbf{k}}(\mathbf{c}, s) \approx \sum_{j=1}^{d+2} K_{j} e^{\lambda_{j}(k) s} \xi_{j}(\mathbf{c})
$$

where the $\left\{K_{j}\right\}_{j=1}^{d+2}$ depend on the initial condition. Note that, as seen in Eqs. (46) and (47), the hydrodynamic eigenfunctions given by Eqs. (48)-(50) expand the subspace of functions generated by the difference of a local $\beta$ state with the stationary state. Then, Eq. (57) can be rewritten in the original variables as

$$
\delta f(\mathbf{r}, \mathbf{v}, t) \approx \frac{n(\mathbf{r}, t)}{v(\mathbf{r}, t)^{d}} \chi\left[\frac{\mathbf{v}-\mathbf{u}(\mathbf{r}, t)}{v(\mathbf{r}, t)}, \frac{\bar{v}_{s}[n(\mathbf{r}, t)]}{v(\mathbf{r}, t)}\right]-f_{s}(v) .
$$

This shows that for small gradients (or, equivalently, for $k \ll 1)$ and in the long time limit, i.e., in the time in which the nonhydrodynamic modes have decayed, all the time dependence in the distribution function is through the hydrodynamic fields. Moreover, the distribution function takes the form of a local $\beta$-state distribution, which plays, in this context, the role of a reference state. To evaluate explicitly the time evolution of the fields, it is necessary to calculate $\left\{\lambda_{j}(k)\right\}_{j=1}^{d+2}$. If we assume that they are analytic in $k$, this can be used to study the eigenvalue problem, Eq. (42), by use of standard perturbation theory, as was performed in Refs. $[8,10]$ for the free-cooling case. In the next section, we will evaluate the eigenvalues to Navier-Stokes order, i.e., $k^{2}$ order, but by use of a different method, deriving the evolution equations for the linear deviations of the hydrodynamic fields.

\section{LINEAR HYDRODYNAMICS AROUND THE STATIONARY STATE}

The objective of this section is to derive evolution equations for the deviations of the hydrodynamic fields around its stationary values at Navier-Stokes order. The analysis of these equations will clarify the differences and analogies with 
respect to the elastic case. The hydrodynamic eigenvalues, $\left\{\lambda_{j}(k)\right\}_{j=1}^{d+2}$, will be obtained by identifying the asymptotic behavior of the solutions in the proper limit. Let us start writing the deviations of the hydrodynamic fields as

$$
\begin{gathered}
\rho(\mathbf{l}, s) \equiv \frac{n(\mathbf{r}, t)-n}{n}=\int d \mathbf{c} \delta \chi(\mathbf{l}, \mathbf{c}, s), \\
\mathbf{w}(\mathbf{l}, s) \equiv \frac{\mathbf{u}(\mathbf{r}, t)}{v_{s}}=\int d \mathbf{c c} \delta \chi(\mathbf{l}, \mathbf{c}, s), \\
\theta(\mathbf{l}, s) \equiv \frac{T(\mathbf{r}, t)-T_{s}}{T_{s}}=\int d \mathbf{c}\left(\frac{2 c^{2}}{d}-1\right) \delta \chi(\mathbf{l}, \mathbf{c}, s),
\end{gathered}
$$

which can be expressed in terms of the scalar products of the distribution function with the functions $\left\{\bar{\xi}_{j}\right\}_{j=1}^{d+2}$. In Fourier space, they read

$$
\begin{gathered}
\left\langle\bar{\xi}_{1}(c) \mid \delta \chi_{\mathbf{k}}(\mathbf{c}, s)\right\rangle=\rho_{\mathbf{k}}(s), \\
\left\langle\bar{\xi}_{2}(\mathbf{c}) \mid \delta \chi_{\mathbf{k}}(\mathbf{c}, s)\right\rangle=\mathbf{w}_{\mathbf{k}}(s), \\
\left\langle\bar{\xi}_{3}(c) \mid \delta \chi_{\mathbf{k}}(\mathbf{c}, s)\right\rangle=\frac{1}{2} \theta_{\mathbf{k}}(s)+\frac{1}{3} \rho_{\mathbf{k}}(s) .
\end{gathered}
$$

Now, let us define the relevant projector in the hydrodynamic subspace,

$$
\mathcal{P} h(\mathbf{c})=\sum_{j=1}^{d+2}\left\langle\bar{\xi}_{j}(\mathbf{c}) \mid h(\mathbf{c})\right\rangle \xi_{j}(\mathbf{c}),
$$

and also the orthogonal one,

$$
\mathcal{Q}=\mathcal{I}-\mathcal{P}
$$

where $\mathcal{I}$ is the identity operator. As alluded to above, $\left\langle\bar{\xi}_{3} \mid h\right\rangle$ is not the actual component of $h$ into $\xi_{3}$ but Eq. (65) still defines a projector as $\mathcal{P}^{2}=\mathcal{I}$ (we also have $\mathcal{Q}^{2}=\mathcal{I}$ ).

If we apply the projectors $\mathcal{P}$ and $\mathcal{Q}$ to the linearized Boltzmann equation (40), we obtain the following set of coupled equations:

$$
\begin{aligned}
& {\left[\frac{\partial}{\partial s}-\mathcal{P}[\Lambda(\mathbf{c})-i \mathbf{k} \cdot \mathbf{c}] \mathcal{P}\right] \mathcal{P} \delta \chi_{\mathbf{k}}(\mathbf{c}, s)} \\
& \quad=[\mathcal{P} \Lambda(\mathbf{c})-\mathcal{P} i \mathbf{k} \cdot \mathbf{c}] \mathcal{Q} \delta \chi_{\mathbf{k}}(\mathbf{c}, s), \\
& {\left[\frac{\partial}{\partial s}-\mathcal{Q}[\Lambda(\mathbf{c})-i \mathbf{k} \cdot \mathbf{c}] \mathcal{Q}\right] \mathcal{Q} \delta \chi_{\mathbf{k}}(\mathbf{c}, s)} \\
& =-\mathcal{Q} i \mathbf{k} \cdot \mathbf{c} \mathcal{P} \delta \chi_{\mathbf{k}}(\mathbf{c}, s),
\end{aligned}
$$

where we have used that $\mathcal{Q} \Lambda \mathcal{P}=0$. However, $\mathcal{P} \Lambda \mathcal{Q} \neq 0$ because $\bar{\xi}_{3}$ is not a left eigenfunction of $\Lambda$. In fact, we have

$$
\mathcal{P} \Lambda(\mathbf{c}) \mathcal{Q} h(\mathbf{c})=\xi_{3}(c)\left\langle\bar{\xi}_{3}(c) \mid \Lambda(\mathbf{c}) \mathcal{Q} h(\mathbf{c})\right\rangle .
$$

The $d+2$ components of Eq. (67) are the evolution equations for the hydrodynamic fields

$$
\begin{gathered}
\frac{\partial}{\partial s} \rho_{\mathbf{k}}(s)+i \mathbf{k} \cdot \mathbf{w}_{\mathbf{k}}(s)=0 \\
\frac{\partial}{\partial s} \mathbf{w}_{\mathbf{k}}(s)+\frac{i}{2} \mathbf{k}\left[\rho_{\mathbf{k}}(s)+\theta_{\mathbf{k}}(s)\right]+i \mathbf{k} \cdot \overleftrightarrow{\Pi}_{\mathbf{k}}(s)=\mathbf{0} \\
\frac{\partial}{\partial s} \theta_{\mathbf{k}}(s)+\gamma\left[\frac{2}{3} \rho_{\mathbf{k}}(s)+\theta_{\mathbf{k}}(s)\right]+\frac{2 i}{d} \mathbf{k} \cdot \mathbf{w}_{\mathbf{k}}(s) \\
+\frac{2 i}{d} \mathbf{k} \cdot \boldsymbol{\phi}_{\mathbf{k}}(s)=\delta \zeta_{\mathbf{k}}(s)
\end{gathered}
$$

where we have introduced the pressure tensor and heat flux

$$
\begin{aligned}
\overleftrightarrow{\Pi}_{\mathbf{k}}(s) & =\int d \mathbf{c} \overleftrightarrow{\Delta}(\mathbf{c}) \mathcal{Q} \delta \chi_{\mathbf{k}}(\mathbf{c}, s) \\
\boldsymbol{\phi}_{\mathbf{k}}(s) & =\int d \mathbf{c} \Sigma(\mathbf{c}) \mathcal{Q} \delta \chi_{\mathbf{k}}(\mathbf{c}, s)
\end{aligned}
$$

with

$$
\Delta_{j p}(\mathbf{c})=c_{j} c_{p}-\frac{c^{2}}{d} \delta_{j p}, \quad \Sigma_{j}(\mathbf{c})=\left(c^{2}-\frac{d+2}{2}\right) c_{j},
$$

and the deviation of the cooling rate

$$
\delta \zeta_{\mathbf{k}}(s)=\int d \mathbf{c} \frac{2 c^{2}}{d} \Lambda(\mathbf{c}) \mathcal{Q} \delta \chi_{\mathbf{k}}(\mathbf{c}, s) .
$$

Let us note that the operator $\mathcal{Q}$ can be skipped in Eq. (73) because, due to symmetry properties, we have

$$
\begin{aligned}
\left\langle\chi_{s}(c) \Delta_{j p}(\mathbf{c}) \mid \xi_{\beta}(\mathbf{c})\right\rangle & =\left\langle\chi_{s}(c) \Sigma_{j}(\mathbf{c}) \mid \xi_{\beta}(\mathbf{c})\right\rangle=0 \\
j, p & =1, \ldots, d, \quad \beta=1, \ldots d+2 .
\end{aligned}
$$

To evaluate the hydrodynamic equations to $k^{2}$ order, we need the fluxes given by Eq. (73) and, consequently, $\delta \chi_{\mathbf{k}}$ to first order in $k$. This is evaluated in Appendix $C$ for an initial condition of the form $\mathcal{Q} \delta \chi_{\mathbf{k}}(\mathbf{c}, 0)=0$ and neglecting all the $k$ contributions in the kinetic modes, yielding

$$
\mathcal{Q} \delta \chi_{\mathbf{k}}(\mathbf{c}, s) \approx-\int_{0}^{s} d s^{\prime} e^{\mathcal{Q} \Lambda(\mathbf{c}) \mathcal{Q}\left(s-s^{\prime}\right)} \mathcal{Q} i \mathbf{k} \cdot \mathbf{c} \mathcal{P} \delta \chi_{\mathbf{k}}\left(\mathbf{c}, s^{\prime}\right) .
$$

Note that as the hydrodynamic eigenfunctions expand the subspace of functions generated by the difference of a local $\beta$ state with the stationary state, the condition $\mathcal{Q} \delta \chi_{\mathbf{k}}(\mathbf{c}, 0)=0$ represents an initial condition of the local $\beta$-state form. For an arbitrary initial condition, there would be an additional initial transient till the distribution function reaches the form given by Eq. (57). When (77) is inserted in Eq. (73), taking into account the symmetries of the system as discussed in Appendix $\mathrm{C}$, the following expressions for the fluxes are obtained,

$$
\begin{aligned}
\Pi_{\mathbf{k}, j p}(s)= & -i \int_{0}^{s} d s^{\prime} G_{x y}\left(s-s^{\prime}\right)\left[k_{j} w_{\mathbf{k}, p}\left(s^{\prime}\right)+k_{p} w_{\mathbf{k}, j}\left(s^{\prime}\right)\right. \\
& \left.-\frac{2}{d} \delta_{j p} \mathbf{k} \cdot \mathbf{w}_{\mathbf{k}}\left(s^{\prime}\right)\right]
\end{aligned}
$$

and

$$
\begin{aligned}
\phi_{\mathbf{k}, j}(s)= & -i k_{j} \int_{0}^{s} d s^{\prime}\left\{\rho_{\mathbf{k}}\left(s^{\prime}\right)\left[H_{1}\left(s-s^{\prime}\right)+\frac{1}{3} H_{3}\left(s-s^{\prime}\right)\right]\right. \\
& \left.+\frac{1}{2} \theta_{\mathbf{k}}\left(s^{\prime}\right) H_{3}\left(s-s^{\prime}\right)\right\},
\end{aligned}
$$

where we have introduced the "correlation" functions,

$$
G_{x y}(s)=\int d \mathbf{c} \Delta_{x y}(\mathbf{c}) e^{\Lambda(\mathbf{c}) s} c_{x} \xi_{2, y}(\mathbf{c})
$$

and

$$
H_{j}(s)=\int d \mathbf{c} \Sigma_{x}(\mathbf{c}) e^{\Lambda(\mathbf{c}) s} c_{x} \xi_{j}(\mathbf{c}), \quad j=1,3 .
$$

It is important to remark that, as $\Delta_{x y}$ and $\Sigma_{x}$ are orthogonal to the hydrodynamic modes, the functions $G_{x y}(s)$ and $H_{j}(s)$ decay with the kinetic modes. Under the same hypothesis as 
for the fluxes, the cooling rate, $\delta \zeta_{\mathbf{k}}$, is evaluated in Appendix D to $k^{2}$ order, with the result

$$
\begin{aligned}
\delta \zeta_{\mathbf{k}}(s)= & -2 i \int_{0}^{s} d s^{\prime} \mathbf{k} \cdot \mathbf{w}_{\mathbf{k}}\left(s^{\prime}\right) Z\left(s-s^{\prime}\right) \\
& -2 k^{2} \int_{0}^{s} d s^{\prime}\left\{\rho_{\mathbf{k}}\left(s^{\prime}\right)\left[Z_{1}\left(s-s^{\prime}\right)+\frac{1}{3} Z_{3}\left(s-s^{\prime}\right)\right]\right. \\
& \left.+\frac{1}{2} \theta_{\mathbf{k}}\left(s^{\prime}\right) Z_{3}\left(s-s^{\prime}\right)\right\}
\end{aligned}
$$

We have introduced here the functions

$$
Z(s)=\left\langle\bar{\xi}_{3}(\mathbf{c}) \mid\left[\Lambda(\mathbf{c})-\lambda_{3}\right] e^{\Lambda(\mathbf{c}) s} c_{x} \xi_{2, x}(\mathbf{c})\right\rangle
$$

and

$$
\begin{aligned}
Z_{j}(s) & =\left\langle\bar{\xi}_{3}(\mathbf{c}) \mid\left[\Lambda(\mathbf{c})-\lambda_{3}\right] \int_{0}^{s} d s^{\prime} e^{\Lambda(\mathbf{c})\left(s-s^{\prime}\right)} c_{x} \mathcal{Q} e^{\Lambda(\mathbf{c}) s^{\prime}} c_{x} \xi_{j}(\mathbf{c})\right\rangle \\
j & =1,3
\end{aligned}
$$

which, due to the biorthogonality condition, Eq. (54), also decay with the kinetic modes. Note that, at variance with the free-cooling case [7], there is here a first order in $k$ contribution to the cooling rate in the low-density limit (for moderate densities the contribution is also present in the free-cooling case $[3,45])$.

It also proves convenient to introduce the parallel and transversal components of the velocity

$$
\begin{aligned}
w_{\mathbf{k}, \|}(s) & =\hat{\mathbf{k}} \cdot \mathbf{w}_{\mathbf{k}}(s), \quad w_{\mathbf{k}, \perp}^{(j)}=\hat{\mathbf{k}}_{\perp}^{(j)} \cdot \mathbf{w}_{\mathbf{k}}(s), \\
j & =1, \ldots, d-1,
\end{aligned}
$$

where $\hat{\mathbf{k}}=\mathbf{k} / k$ is a unit vector in the direction of $\mathbf{k}$ and $\left\{\hat{\mathbf{k}}_{\perp}^{(j)}\right\}_{j=1}^{d-1}$ is an orthogonal basis of the subspace orthogonal to k. In terms of these components, the hydrodynamic equations are

$$
\begin{gathered}
\frac{\partial}{\partial s} \rho_{\mathbf{k}}(s)+i k w_{\mathbf{k}, \|}(s)=0 \\
\frac{\partial}{\partial s} w_{\mathbf{k}, \perp}^{(j)}(s)+k^{2} \int_{0}^{s} d s^{\prime} G_{x y}\left(s-s^{\prime}\right) w_{\mathbf{k}, \perp}^{(j)}\left(s^{\prime}\right)=0 \\
j=1, \ldots, d-1, \\
\frac{\partial}{\partial s} w_{\mathbf{k}, \|}(s)+\frac{i}{2} k\left[\rho_{\mathbf{k}}(s)+\theta_{\mathbf{k}}(s)\right]+2 \frac{d-1}{d} k^{2} \\
\times \int_{0}^{s} d s^{\prime} G_{x y}\left(s-s^{\prime}\right) w_{\mathbf{k}, \|}\left(s^{\prime}\right)=0 \\
\frac{\partial}{\partial s} \theta_{\mathbf{k}}(s)+\gamma\left[\frac{2}{3} \rho_{\mathbf{k}}(s)+\theta_{\mathbf{k}}(s)\right]+\frac{2 i}{d} k w_{\mathbf{k}, \|}(s) \\
+2 i k \int_{0}^{s} d s^{\prime} Z\left(s-s^{\prime}\right) w_{\mathbf{k}, \|}\left(s^{\prime}\right) \\
+\frac{2}{d} k^{2} \int_{0}^{s} d s^{\prime}\left[G_{1}\left(s-s^{\prime}\right) \rho_{\mathbf{k}}\left(s^{\prime}\right)+G_{3}\left(s-s^{\prime}\right) \theta_{\mathbf{k}}\left(s^{\prime}\right)\right]=0,
\end{gathered}
$$

where we have introduced the new correlation functions

$$
\begin{aligned}
& G_{1}(s)=H_{1}(s)+\frac{1}{3} H_{3}(s)+d Z_{1}(s)+\frac{d}{3} Z_{3}(s), \\
& G_{3}(s)=\frac{1}{2} H_{3}(s)+\frac{d}{2} Z_{3}(s) .
\end{aligned}
$$

It is noteworthy that, apart from the assumption of an initial condition in the hydrodynamic subspace, the only approximations made in the derivation of Eqs. (86)-(89) are the expansion to second order in the gradients of the hydrodynamic fields and the neglect of the $k$ contribution in the memory kernels that all decay with the kinetic modes. Of course, the kernels are for the moment unknown, but we will see later that they can be calculated approximately. Before doing so, we evaluate the asymptotic behavior of Eqs. (86)-(89) in the hydrodynamic limit [under which Eq. (57) was derived].

Due to the structure of the equations, it is convenient to introduce the Laplace transforms of the fields,

$$
\bar{f}(z)=\int_{0}^{\infty} d s e^{-z s} f(s), \quad f(s)=\frac{1}{2 \pi i} \int_{c-i \infty}^{c+i \infty} d z e^{z s} \bar{f}(z)
$$

where $c$ is bigger than the real part of all the poles of $\bar{f}$. In the Laplace space the convolutions transform into products and Eqs. (86)-(89) become

$$
\begin{gathered}
z \bar{w}_{\mathbf{k}, \perp}^{(j)}(z)+k^{2} \bar{G}_{x y}(z) \bar{w}_{\mathbf{k}, \perp}^{(j)}(z)=w_{\mathbf{k}, \perp}^{(j)}(0), \quad j=1, \ldots, d-1 . \\
{[z I+A(k, z)]\left(\begin{array}{c}
\bar{\rho}_{\mathbf{k}}(z) \\
\bar{w}_{\mathbf{k}, \|}(z) \\
\bar{\theta}_{\mathbf{k}}(z)
\end{array}\right)=\left(\begin{array}{c}
\rho_{\mathbf{k}}(0) \\
w_{\mathbf{k}, \|}(0) \\
\theta_{\mathbf{k}}(0)
\end{array}\right),}
\end{gathered}
$$

where we have introduced the matrix

$$
\begin{aligned}
& A(k, z) \\
& \quad=\left(\begin{array}{ccc}
0 & i k & 0 \\
\frac{i}{2} k & 2 \frac{d-1}{d} \bar{G}_{x y}(z) k^{2} & \frac{i}{2} k \\
\frac{2}{3} \gamma+\frac{2}{d} \bar{G}_{1}(z) k^{2} & i \bar{q}(z) k & \gamma+\frac{2}{d} \bar{G}_{3}(z) k^{2}
\end{array}\right),
\end{aligned}
$$

with

$$
\bar{q}(z)=\frac{2}{d}[1+d \bar{Z}(z)] .
$$

The equation for the transverse velocity, Eq. (92), is analyzed in detail in Appendix E. Assuming that $G_{x y}(s)$ is a linear combination of kinetic modes (as is expected to be), we obtain in the hydrodynamic limit

$$
w_{\mathbf{k}, \perp}^{(j)}(s) \approx w_{\mathbf{k}, \perp}^{(j)}(0) e^{-\eta k^{2} s},
$$

where we have introduced the shear viscosity

$$
\eta=\int_{0}^{\infty} d s G_{x y}(s) .
$$

In the same limit, the solution of the coupled hydrodynamic equation (93), is analyzed in Appendix F, obtaining

$$
|y(\mathbf{k}, s)\rangle \approx \sum_{\beta=1}^{3}\left\langle\phi_{\beta} \mid y(\mathbf{k}, 0)\right\rangle e^{\lambda_{\beta}(k) s}\left|\psi_{\beta}^{(0)}\right\rangle,
$$

where we have introduced the notation

$$
|y(\mathbf{k}, s)\rangle \equiv\left(\begin{array}{c}
\rho_{\mathbf{k}}(s) \\
w_{\mathbf{k}, \|}(s) \\
\theta_{\mathbf{k}}(s)
\end{array}\right) .
$$


The functions $\left\{\left|\psi_{\beta}^{(0)}\right\rangle\right\}_{\beta=1}^{3}$ are the zeroth order in $k$ contribution to the expansion of the eigenfunctions, $\left|\psi_{\beta}(k, z)\right\rangle$, of the matrix $A(k, z)$

$$
\left|\psi_{\beta}(k, z)\right\rangle=\left|\psi_{\beta}^{(0)}(z)\right\rangle+k\left|\psi_{\beta}^{(1)}(z)\right\rangle+k^{2}\left|\psi_{\beta}^{(2)}(z)\right\rangle+\cdots,
$$

with $A(k, z)\left|\psi_{\beta}(k, z)\right\rangle=a_{\beta}(k, z)\left|\psi_{\beta}(k, z)\right\rangle$. They are calculated in Appendix F obtaining

$$
\left|\psi_{1}^{(0)}\right\rangle=\left(\begin{array}{c}
-6 \\
\sqrt{6} \\
4
\end{array}\right), \quad\left|\psi_{2}^{(0)}\right\rangle=\left(\begin{array}{c}
6 \\
\sqrt{6} \\
-4
\end{array}\right), \quad\left|\psi_{3}^{(0)}\right\rangle=\left(\begin{array}{l}
0 \\
0 \\
1
\end{array}\right)
$$

where we see that they do not depend on $z$. The other set of functions, $\left\{\left\langle\phi_{\beta}\right|\right\}_{\beta=1}^{3}$, is the biorthogonal set

$$
\begin{aligned}
\left\langle\phi_{1}\right| & =\left(-\frac{1}{12}, \frac{1}{2 \sqrt{6}}, 0\right), \\
\left\langle\phi_{2}\right| & =\left(\frac{1}{12}, \frac{1}{2 \sqrt{6}}, 0\right), \\
\left\langle\phi_{3}\right| & =\left(\frac{2}{3}, 0,1\right),
\end{aligned}
$$

which is constructed to have $\left\langle\phi_{\beta} \mid \psi_{\beta^{\prime}}^{(0)}\right\rangle=\delta_{\beta, \beta^{\prime}}$. The scalar product $\langle u \mid v\rangle$ between two vectors $|u\rangle$ and $|v\rangle$ is the usual Euclidean scalar product. There is no confusion with the one introduced in Eq. (43) because they appear in different contexts. The explicit expressions of the hydrodynamic eigenvalues, $\left\{\lambda_{\beta}(k)\right\}_{\beta=1}^{3}$, to $k^{2}$ order are

$$
\begin{gathered}
\lambda_{1}(k)=+\frac{i}{\sqrt{6}} k-\left[\frac{d-1}{d} \eta+\frac{1}{4 \gamma}\left(\frac{2}{3}+q_{0}\right)\right] k^{2}, \\
\lambda_{2}(k)=-\frac{i}{\sqrt{6}} k-\left[\frac{d-1}{d} \eta+\frac{1}{4 \gamma}\left(\frac{2}{3}+q_{0}\right)\right] k^{2}, \\
\lambda_{3}(k)=-\gamma+\left[\frac{1}{3 \gamma}+\frac{1}{2 \gamma} q_{w}-\frac{2}{d}\left(\tilde{\kappa}+\zeta_{\theta}\right)\right] k^{2},
\end{gathered}
$$

where the shear viscosity, $\eta$, is given in Eq. (97), and we have introduced the heat conductivity, $\widetilde{\kappa}$, as

$$
\tilde{\kappa}=\frac{1}{2} \int_{0}^{\infty} d s e^{\gamma s} H_{3}(s) .
$$

The expressions for the other transport coefficients are

$$
\begin{gathered}
q_{0}=\frac{2}{d}+2 \int_{0}^{\infty} d s Z(s), \\
q_{w}=\frac{2}{d}+2 \int_{0}^{\infty} d s e^{\gamma s} Z(s), \\
\zeta_{\theta}=\frac{d}{2} \int_{0}^{\infty} d s e^{\gamma s} Z_{3}(s) .
\end{gathered}
$$

Equations (96) and (98) with the expressions for the transport coefficients, Eqs. (97) and (106)-(109), are the main results of the paper. It is important to remark that the equations are valid in the linear regime close to the stationary state, but the transport coefficients depend on the structure of the nonstationary $\beta$ state through the eigenvalue $\gamma$ and the third eigenfunction. Actually, similar effects are shown to be essential to understand the dynamics of a homogeneous perturbation of the temperature close to the steady uniform shear flow for granular gases [46]. Let us also note that, although there is a coupling between the heat flux and the density as it is seen in Eq. (79), this coupling is not reflected at the level of Navier-Stokes linear hydrodynamic [there is no contribution from $H_{1}(s)$ in the transport coefficients]. This is also the case in the free-cooling case where the hydrodynamic eigenvalues to $k^{2}$ order do not depend on the diffusive conductivity $[8,10]$ (the coupling is expected to appear beyond $k^{2}$ order). Moreover, the memory kernel $Z(s)$ appears in $q_{0}$ and $q_{w}$ weighted in different ways, reflecting the non-Markovian character of Eqs. (86)-(89). Nevertheless, as we shall see in the remainder, these effects are expected to be small. We also emphasize that the viscosity and heat conductivity have been calculated in Ref. [37] applying the Chapman-Enskog expansion to the inelastic Enskog equation, obtaining equivalent expressions for both transport coefficients in the low-density limit. This can be seen by performing formally the time integral in the Green-Kubo expressions for the transport coefficients, Eqs. (97)-(106).

Our goal is now to calculate all the correlations functions, $G_{x y}(s), G_{1}(s), G_{3}(s)$, and $Z(s)$, that appear in the hydrodynamic equations, Eqs. (86)-(89), in an approximate way. With this, we will be able to obtain explicit formulas for all the transport coefficients. The idea is reminiscent of that used for free-cooling systems [47] and consists in treating the functions $\bar{\xi}_{3}(c), \chi_{s}(c) \Delta_{j p}(\mathbf{c})$, and $\chi_{s}(c) \Sigma_{j}(\mathbf{c})$ as if they were eigenfunctions of the adjoint linearized Boltzmann operator, $\Lambda^{+}$[the adjoint is taken with the scalar product of Eq. (43)]. That is, we assume

$$
\Lambda^{+}(\mathbf{c}) \bar{\xi}_{3}(c) \approx \lambda_{3} \bar{\xi}_{3}(c)
$$

and

$$
\begin{aligned}
\Lambda^{+}(\mathbf{c}) \chi_{s}(c) \Delta_{j p}(\mathbf{c}) & \approx \lambda_{\mathrm{NH}}^{(1)} \chi_{s}(c) \Delta_{j p}(\mathbf{c}), \\
\Lambda^{+}(\mathbf{c}) \chi_{s}(c) \Sigma_{j}(\mathbf{c}) & \approx \lambda_{\mathrm{NH}}^{(2)} \chi_{s}(c) \Sigma_{j}(\mathbf{c}),
\end{aligned}
$$

where $\lambda_{\mathrm{NH}}^{(1)}$ and $\lambda_{\mathrm{NH}}^{(2)}$ are two nonhydrodynamic (kinetic) eigenvalues that have to be calculated consistently. Within this approximation we trivially have

$$
Z(s) \approx 0, \quad Z_{j}(s) \approx 0, \quad j=1,3,
$$

so

$$
q_{0} \approx \frac{2}{d}, \quad q_{w} \approx \frac{2}{d}, \quad \zeta_{\theta} \approx 0,
$$

and

$$
G_{1}(s) \approx H_{1}(s)+\frac{1}{3} H_{3}(s), \quad G_{3}(s) \approx \frac{1}{2} H_{3}(s) .
$$

Assuming the property (111) holds, the functions $G_{x y}(s)$, $H_{1}(s)$, and $H_{3}(s)$ follow, by straightforward calculations,

$$
\begin{aligned}
G_{x y}(s) & =\frac{1}{2} e^{\lambda_{\mathrm{NH}}^{(1)} s}, \quad H_{1}(s)=-\frac{(d+2)\left(2+a_{2}^{s}\right)}{12} e^{\lambda_{\mathrm{NH}}^{(2)} s}, \\
H_{3}(s) & =\left[(d+2)\left(\frac{1}{2}+a_{2}^{s}\right)-\left.\frac{d+2}{4} \frac{d a_{2}(\beta)}{d \beta}\right|_{\beta=1}\right] e^{\lambda_{\mathrm{NH}}^{(2)} s},
\end{aligned}
$$



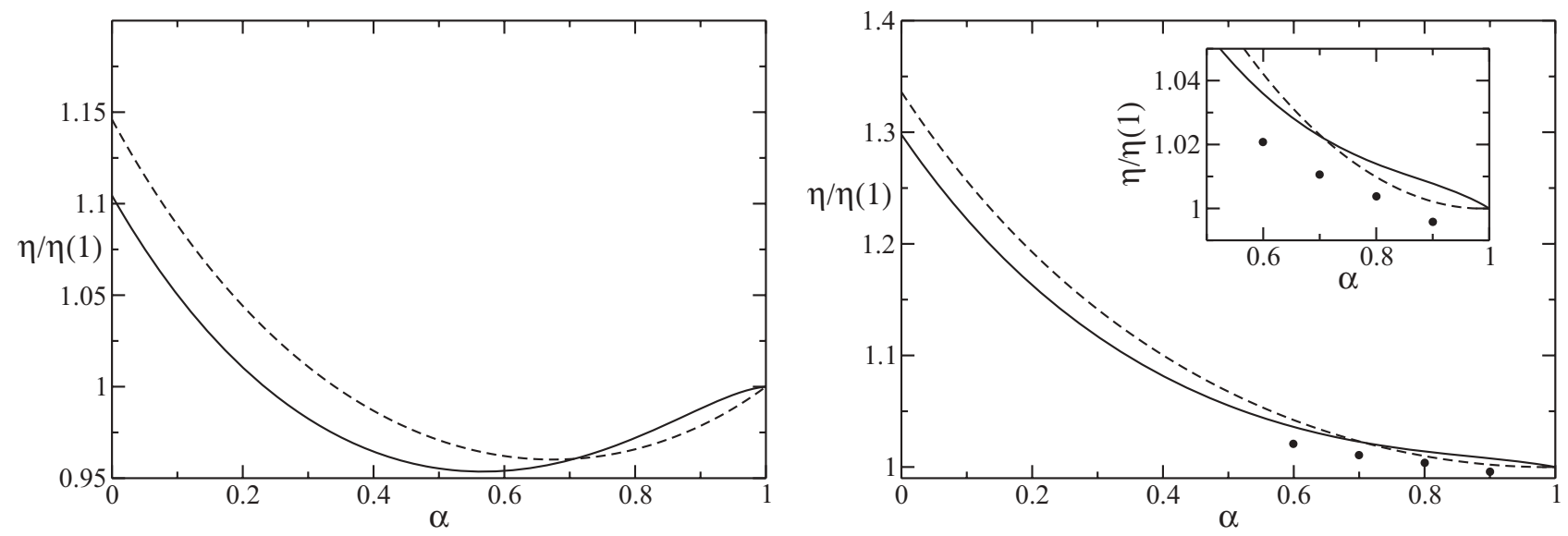

FIG. 1. Reduced viscosity of a two-dimensional system (left) and three-dimensional system (right) as a function of the inelasticity. The solid line is the theoretical prediction given by Eq. (117), the dashed line is the theoretical prediction of Ref. [29], and the dots are the simulation results of Ref. [29].

where the eigenvalues $\lambda_{\mathrm{NH}}^{(1)}$ and $\lambda_{\mathrm{NH}}^{(2)}$ are calculated in Appendix $G$ in the approximation of Eq. (111). With these functions, the transport coefficient are easily calculated,

$$
\begin{aligned}
& \eta=\frac{1}{2\left|\lambda_{\mathrm{NH}}^{(1)}\right|}, \\
& \widetilde{\kappa}=\frac{1}{2\left(\left|\lambda_{\mathrm{NH}}^{(2)}\right|-\gamma\right)}\left[(d+2)\left(\frac{1}{2}+a_{2}^{s}\right)-\left.\frac{d+2}{4} \frac{d a_{2}(\beta)}{d \beta}\right|_{\beta=1}\right],
\end{aligned}
$$

where, as shown in Appendix A, the contribution coming from $\left.\frac{d a_{2}(\beta)}{d \beta}\right|_{\beta=1}$ is of the same order as the one coming from $a_{2}^{s}$. Let us note that, as the approximation (110) and (111) implies that $\zeta_{\theta}=0$ and kills the difference between $q_{0}$ and $q_{w}$, our results agree with the ones of Garzó et al. [37] when our thermostat is considered and the low-density limit is taken. The only difference is the explicit approximate expressions for $\eta$ and $\tilde{\kappa}$ (although, as said, the exact expressions for both transport coefficients are the same).

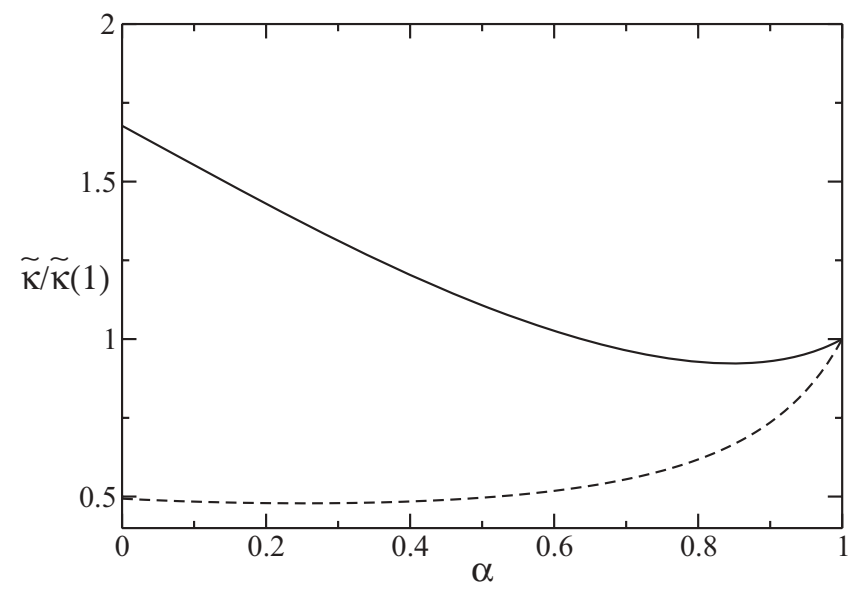

In Fig. 1 the reduced viscosity $\frac{\eta(\alpha)}{\eta(1)}$ is plotted as a function of the inelasticity for $d=2$ and $d=3$, and in Fig. 2 the same is done but for the reduced conductivity $\frac{\widetilde{\kappa}(\alpha)}{\widetilde{\kappa}(1)}$. Although strictly speaking, we do not consider the exact same thermostating mechanism as in Ref. [29] where the driving amplitude is chosen-for convenience-to depend on the local temperature, it is nevertheless relevant to compare our predictions to those of Ref. [29]. In the case of the shear viscosity, we obtain exactly the same result. The differences in Fig. 1 are due to the approximate method to evaluate the coefficient, yielding a smoother curve with the present method. In contrast, for the reduced conductivity, we obtain strong discrepancies. Equation (117) predicts an enhanced of the conductivity as the inelasticity increases while the prediction of Ref. [29] goes in the opposite direction. For completeness, we have reported some simulation data available in the literature. For the shear viscosity, the results were taken from Ref. [29] for $d=3$. The agreement with the theoretical prediction is good. For the heat conductivity, we have taken the results of Ref. [35], pertaining

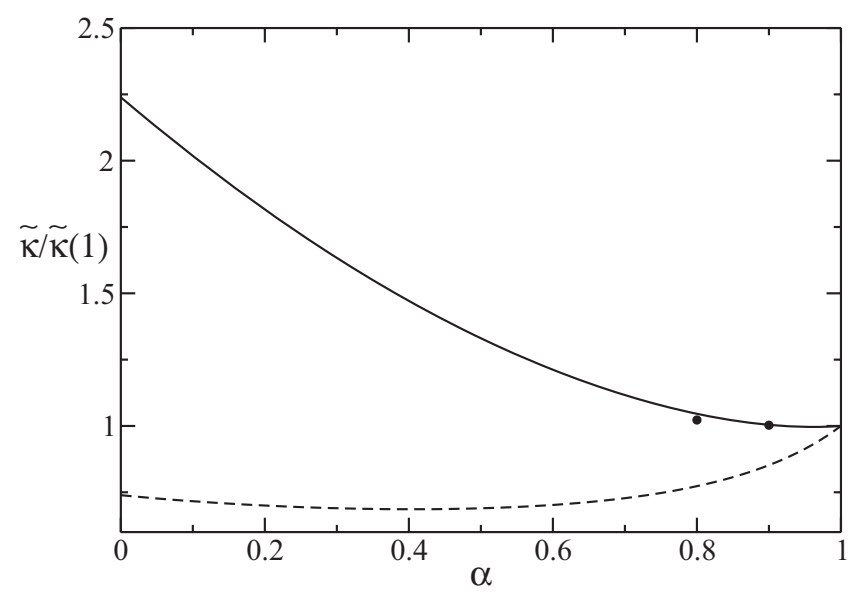

FIG. 2. Reduced conductivity of a two-dimensional system (left) and three-dimensional system (right) as a function of the inelasticity. The solid line is the theoretical prediction given by Eq. (117), the dashed line is the theoretical prediction of Ref. [29], and the dots are the simulation results of Ref. [35]. 
also to dissipative hard spheres for the smallest density and wave vector. The agreement with equation (117) in this case is very good for the two values of the coefficient of normal restitution. Let us note that the transport coefficients were also evaluated for moderate densities in Ref. [48] via the Enskog equation and the density dependence agreed qualitatively well with the simulation results of Ref. [35].

\section{CONCLUSIONS AND PERSPECTIVES}

In this paper we have derived the Navier-Stokes hydrodynamic equations for a system of hard particles heated by the so-called stochastic thermostat. We have restricted the study to situations close to the homogeneous stationary state that the system reaches in the long time limit. Under these conditions, the system is described by the Boltzmann equation linearized around the stationary state. We could calculate the eigenvalues and eigenfunctions of the operator describing the dynamics of the system that are relevant in the hydrodynamic description. Let us remark that, although we are considering linear response around the stationary state, the modes depend on the properties of the time-dependent $\beta$ state through quantities related to $\left.\frac{\partial \chi(c, \beta)}{\partial \beta}\right|_{\beta=1}$. The properties of the $\beta$ state brought to the fore were summarized in Sec. II and in particular, $\beta-1$ can be viewed as measuring the distance to stationarity. With the aid of these modes, we derived the linear Navier-Stokes equations obtaining formulas for the transport coefficients that are expressed as Green-Kubo relations. Assuming that the time correlation functions that appear in these formulas decay with only one kinetic mode, we calculated explicitly the transport coefficients as functions of the inelasticity, $\alpha$, and the spatial dimension, $d$. Let us note that, at this level, the dynamics also depends on the properties of the $\beta$ state through the transport coefficients. Moreover, as it is reflected in Eq. (58), the $\beta$ state plays the role of reference state in the sense that, in the hydrodynamic scale a local $\beta$-state distribution is reached. This fact is in connection with the results of Ref. [15] where it is seen that, close to a stationary state, the zeroth order in the gradients distribution is not merely the local stationary distribution but a more complex one (in our case played by the $\beta$ state). In this sense, for situations where nonlinear effects are important, the complete hydrodynamic equations at Navier-Stokes order could be derived by the Chapman-Enskog scheme taking into account these ideas. We hope this work will contribute to stimulate more studies in this direction and also at the level of computer simulations by, for example, measuring the transport coefficients. Also, since many effects shown in the paper depend on the two-parameter distribution of the $\beta$ state, it is expected that similar phenomena will occur for other sorts of homogeneous thermostats.

\section{ACKNOWLEDGMENTS}

We thank Vicente Garzó and Moisés García Chamorro for useful discussions. This research was supported by the Ministerio de Educación y Ciencia (Spain) through Grant No. FIS2011-24460 (partially financed by FEDER funds).

\section{APPENDIX A: EVALUATION OF $\boldsymbol{\gamma}$ IN THE FIRST SONINE APPROXIMATION}

In this Appendix we calculate the coefficient $\gamma$ given by Eq. (28),

$$
\gamma=3 \mu(1)-\left.\frac{d \mu(\beta)}{d \beta}\right|_{\beta=1}
$$

in the first Sonine approximation. By substituting the approximate expression of $\chi(c, \beta)$ given by Eq. (16) into the definition of $\mu(\beta)$ [see Eq. (24)], we obtain

$$
\mu(\beta)=\mu_{M}+\mu_{S} a_{2}(\beta),
$$

where

$$
\mu_{M}=-\frac{1}{2 d} \int d \mathbf{c}_{1} \int d \mathbf{c}_{2} \chi_{M}\left(c_{1}\right) \chi_{M}\left(c_{2}\right) T_{0}\left(\mathbf{c}_{1}, \mathbf{c}_{2}\right)\left(c_{1}^{2}+c_{2}^{2}\right)
$$

and

$$
\begin{aligned}
\mu_{S}= & -\frac{1}{d} \int d \mathbf{c}_{1} \int d \mathbf{c}_{2} \chi_{M}\left(c_{1}\right) \chi_{M}\left(c_{2}\right) S_{2}\left(c_{1}^{2}\right) \\
& \times T_{0}\left(\mathbf{c}_{1}, \mathbf{c}_{2}\right)\left(c_{1}^{2}+c_{2}^{2}\right) .
\end{aligned}
$$

The integrals can be readily performed with the result

$$
\mu_{M}=\frac{\pi^{\frac{d-1}{2}}\left(1-\alpha^{2}\right)}{\sqrt{2} d \Gamma(d / 2)}, \quad \mu_{S}=\frac{3 \pi^{\frac{d-1}{2}}\left(1-\alpha^{2}\right)}{16 \sqrt{2} d \Gamma(d / 2)} .
$$

Finally, the expression for $\gamma$ is

$$
\gamma=3 \mu_{M}+\left[3 a_{2}(1)-\left.\frac{d a_{2}(\beta)}{d \beta}\right|_{\beta=1}\right] \mu_{S},
$$

where the expression of $a_{2}(\beta)$ has been provided in the main text. In Fig. 3, $\gamma$ is plotted as a function of the inelasticity. The approximate expression of the Ref. [25], $\gamma_{v N}=3 \mu(1)$, is also plotted, finding very similar results. Nevertheless, let us note that, as shown in Fig. 4, the difference between the two expressions, $\gamma_{v N}-\gamma=\left.\frac{d a_{2}(\beta)}{d \beta}\right|_{\beta=1} \mu_{S}$, is of the order of $a_{2}(1)$.

\section{APPENDIX B: EVALUATION OF THE HYDRODYNAMIC EIGENFUNCTIONS}

Let us evaluate the expression

$$
\delta f(\mathbf{v}, t)=\frac{\bar{n}}{\bar{v}_{H}(t)^{d}} \chi\left[\frac{\mathbf{v}}{\bar{v}_{H}(t)}, \frac{\bar{v}_{s}}{\bar{v}_{H}(t)}\right]-f_{s}(v)
$$

to linear order in $\delta n=\bar{n}-n$ and $\delta v_{H}(0)=\bar{v}_{H}(0)-v_{s}$. We consider the case $\mathbf{u}=\mathbf{0}$, for assuming $\mathbf{u} \neq \mathbf{0}$ is a straightforward generalization. Let us rewrite Eq. (B1) as

$$
\begin{aligned}
\delta f(\mathbf{v}, t)= & \frac{\bar{n}}{\bar{v}_{H}(t)^{d}} \chi\left[\frac{\mathbf{v}}{\bar{v}_{H}(t)}, \frac{\bar{v}_{s}}{\bar{v}_{H}(t)}\right]-\frac{\bar{n}}{\bar{v}_{s}^{d}} \chi_{s}\left(\frac{\mathbf{v}}{\bar{v}_{s}}\right) \\
& +\frac{\bar{n}}{\bar{v}_{s}^{d}} \chi_{s}\left(\frac{\mathbf{v}}{\bar{v}_{s}}\right)-f_{s}(v) .
\end{aligned}
$$

The first two terms on the left-hand side of the equation then are the difference between the $\beta$ state and its corresponding stationary state (both are linked to the same density, $\bar{n}$ ), while the last two terms are just the difference between two very 

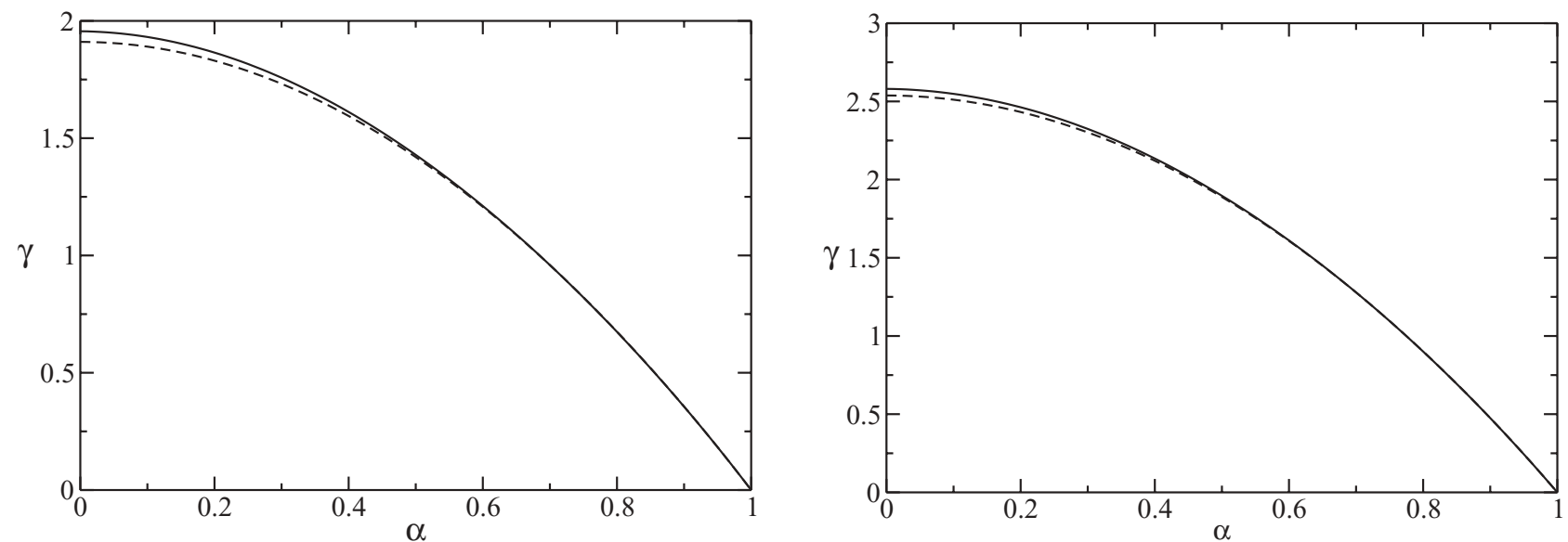

FIG. 3. Eigenvalue $\gamma$ as a function of the inelasticity for a two-dimensional system (left) and three-dimensional system (right). The solid line is the theoretical prediction for $\gamma$ and the dashed lined is the one of Ref. [25], $\gamma_{v N}$.

close stationary states. For the last two terms and, taking into account that

$$
n v_{s}^{3}=\bar{n} \bar{v}_{s}^{3}=\mathcal{K}(\alpha),
$$

as follows from from Eq. (13) which defines the unspecified function $\mathcal{K}$ and, thus,

$$
\begin{aligned}
& \frac{\bar{n}}{\bar{v}_{s}^{d}} \chi_{s}\left(\frac{\mathbf{v}}{\bar{v}_{s}}\right)-\frac{n}{v_{s}^{d}} \chi_{s}\left(\frac{\mathbf{v}}{v_{s}}\right) \\
& \quad=\frac{\mathcal{K}}{\bar{v}_{s}^{d+3}} \chi_{s}\left(\frac{\mathbf{v}}{\bar{v}_{s}}\right)-\frac{\mathcal{K}}{v_{s}^{d+3}} \chi_{s}\left(\frac{\mathbf{v}}{v_{s}}\right) \\
& \quad \approx \mathcal{K} \delta v_{s}\left[-\frac{(d+3)}{v_{s}^{d+4}} \chi_{s}(c)+\frac{1}{v_{s}^{d+3}} \frac{\partial}{\partial v_{s}} \chi_{s}\left(\frac{\mathbf{v}}{v_{s}}\right)\right],
\end{aligned}
$$

where $\delta v_{s}=\bar{v}_{s}-v_{s}$. It is more convenient to write this expression in terms of the difference in densities. Due to Eq. (B3), we have

$$
\frac{\delta n}{n}=-3 \frac{\delta v_{s}}{v_{s}},
$$

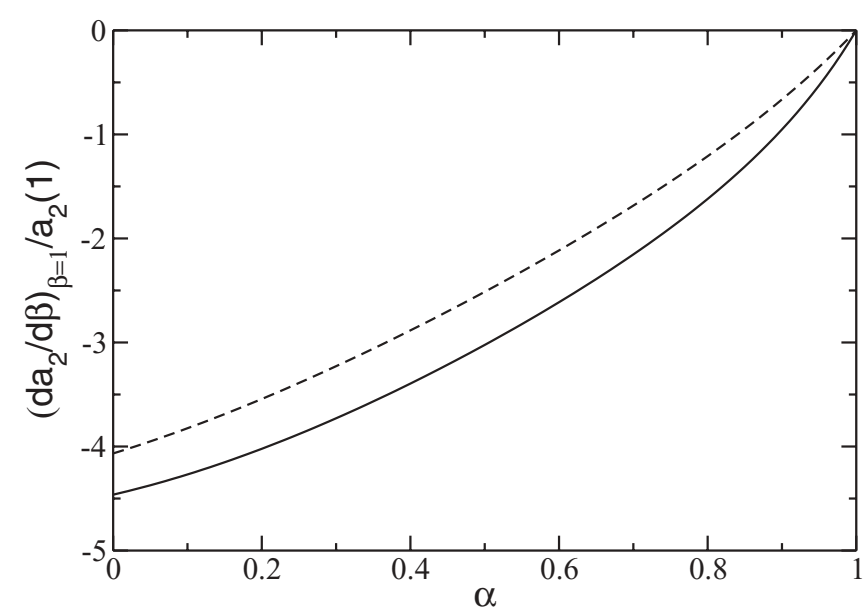

FIG. 4. Quotient between $\left.\frac{d a_{2}(\beta)}{d \beta}\right|_{\beta=1}$ and $a_{2}$ (1) as a function of the inelasticity for $d=2$ (solid line) and $d=3$ (dashed line). Note that $a_{2}(1)=a_{2}^{s}$. so

$$
\begin{aligned}
& \frac{\bar{n}}{\bar{v}_{s}^{d}} \chi_{s}\left(\frac{\mathbf{v}}{\bar{v}_{s}}\right)-\frac{n}{v_{s}^{d}} \chi_{s}\left(\frac{\mathbf{v}}{v_{s}}\right) \\
& \quad \approx \frac{n}{v_{s}^{d}} \frac{\delta n}{n}\left\{\chi_{s}(c)+\frac{1}{3} \frac{\partial}{\partial \mathbf{c}} \cdot\left[\mathbf{c} \chi_{s}(c)\right]\right\} .
\end{aligned}
$$

Now, let us evaluate the other difference,

$$
\begin{aligned}
& \frac{\bar{n}}{\bar{v}_{H}(t)^{d}} \chi\left[\frac{\mathbf{v}}{\bar{v}_{H}(t)}, \frac{\bar{v}_{s}}{\bar{v}_{H}(t)}\right]-\frac{\bar{n}}{\bar{v}_{s}^{d}} \chi_{s}\left(\frac{\mathbf{v}}{\bar{v}_{s}}\right) \\
& \approx \frac{\partial}{\partial v_{H}(0)}\left\{\frac{n}{v_{H}(t)^{d}} \chi\left[\frac{\mathbf{v}}{v_{H}(t)}, \frac{v_{s}}{v_{H}(t)}\right]\right\}_{s}\left[\bar{v}_{H}(0)-\bar{v}_{s}\right],
\end{aligned}
$$

where the subscript $s$ refers to the functional in the stationary state, i.e., $F\left[n, v_{H}(0)\right]_{s}=F\left[\bar{n}, \bar{v}_{s}\right]$. The functional derivative is

$$
\begin{aligned}
& \frac{\partial}{\partial v_{H}(0)}\left\{\frac{n}{v_{H}(t)^{d}} \chi\left[\frac{\mathbf{v}}{v_{H}(t)}, \frac{v_{s}}{v_{H}(t)}\right]\right\} \\
& =-\frac{n}{v_{H}(t)^{d+1}}\left\{\frac{\partial}{\partial \mathbf{c}} \cdot[\mathbf{c} \chi(c, \beta)]+\beta \frac{\partial}{\partial \beta} \chi(c, \beta)\right\} \frac{\partial v_{H}(t)}{\partial v_{H}(0)}
\end{aligned}
$$

while the partial derivative can be calculated, taking into account Eq. (29), giving

$$
\left[\frac{\partial v_{H}(t)}{\partial v_{H}(0)}\right]_{s}=e^{-\gamma \frac{\bar{v}_{s}}{\ell} t} .
$$

Then, by substituting Eq. (B8) into Eq. (B7) and defining $\widetilde{\mathbf{c}}=\mathbf{v} / \bar{v}_{s}$, we have

$$
\begin{aligned}
& \frac{\bar{n}}{\bar{v}_{H}(t)^{d}} \chi\left[\frac{\mathbf{v}}{\bar{v}_{H}(t)}, \frac{\bar{v}_{s}}{\bar{v}_{H}(t)}\right]-\frac{\bar{n}}{\bar{v}_{s}^{d}} \chi_{s}\left(\frac{\mathbf{v}}{\bar{v}_{s}}\right) \\
& \approx-\frac{\bar{n}}{\bar{v}_{s}^{d}}\left\{\frac{\partial}{\partial \widetilde{\mathbf{c}}} \cdot\left[\widetilde{\mathbf{c}} \chi_{s}(\widetilde{c})\right]+\left.\frac{\partial}{\partial \beta} \chi(\widetilde{c}, \beta)\right|_{\beta=1}\right\} \\
& \quad \times e^{-\gamma \frac{\bar{v}_{s}}{\ell} t} \frac{\bar{v}_{H}(0)-\bar{v}_{s}}{\bar{v}_{s}}
\end{aligned}
$$




$$
\begin{aligned}
\approx & -\frac{n}{v_{s}^{d}}\left\{\frac{\partial}{\partial \mathbf{c}} \cdot\left[\mathbf{c} \chi_{s}(c)\right]+\left.\frac{\partial}{\partial \beta} \chi(c, \beta)\right|_{\beta=1}\right\} \\
& \times e^{-\gamma \frac{v_{s}}{\ell} t}\left[\frac{\delta v_{H}(0)}{v_{s}}+\frac{1}{3} \frac{\delta n}{n}\right],
\end{aligned}
$$

where we have substituted $\bar{n}$ by $n$ and $\bar{v}_{s}$ by $v_{s}$ (that can be done to linear order) and we have used that

$$
\frac{\bar{v}_{H}(0)-v_{s}+v_{s}-\bar{v}_{s}}{v_{s}}=\frac{\delta v_{H}(0)}{v_{s}}+\frac{1}{3} \frac{\delta n}{n} .
$$

Taking into account (B6) and (B10), we finally arrive at the result reported in the main text for $\mathbf{u}=\mathbf{0}$,

$$
\begin{aligned}
\delta \chi & (\mathbf{c}, s) \\
= & \frac{\delta n}{n}\left[\chi_{s}(c)+\frac{1}{3} \frac{\partial}{\partial \mathbf{c}} \cdot\left[\mathbf{c} \chi_{s}(c)\right]\right]-\left[\frac{\delta n}{3 n}+\frac{\delta v_{H}(0)}{v_{s}}\right] e^{-\gamma s} \\
& \times\left[\frac{\partial}{\partial \mathbf{c}} \cdot\left[\mathbf{c} \chi_{s}(c)\right]+\left.\frac{\partial}{\partial \beta} \chi(c, \beta)\right|_{\beta=1}\right] .
\end{aligned}
$$

\section{APPENDIX C: EVALUATION OF THE FLUXES TO FIRST ORDER IN $k$}

In this Appendix, we evaluate the fluxes given by Eq. (73) to first order in $k$. The function $\mathcal{Q} \delta \chi_{\mathbf{k}}$ fulfills Eq. (68) and can be integrated formally as

$$
\begin{aligned}
\mathcal{Q} \delta \chi_{\mathbf{k}}(\mathbf{c}, s)= & e^{\mathcal{Q}(\Lambda-i \mathbf{k} \cdot \mathbf{c}) s} \mathcal{Q} \delta \chi_{\mathbf{k}}(\mathbf{c}, 0) \\
& -\int_{0}^{s} d s^{\prime} e^{\mathcal{Q}(\Lambda-i \mathbf{k} \cdot \mathbf{c}) \mathcal{Q}\left(s-s^{\prime}\right)} \mathcal{Q} i \mathbf{k} \cdot \mathbf{c} \mathcal{P} \delta \chi_{\mathbf{k}}\left(\mathbf{c}, s^{\prime}\right) .
\end{aligned}
$$

Choosing the initial condition in the hydrodynamic subspace, i.e., $\mathcal{Q} \delta \chi_{\mathbf{k}}(\mathbf{c}, 0)=0$, and neglecting the $k$ contribution in the kinetic modes, we have

$$
\mathcal{Q} \delta \chi_{\mathbf{k}}(\mathbf{c}, s) \approx-\int_{0}^{s} d s^{\prime} e^{\mathcal{Q} \Lambda \mathcal{Q}\left(s-s^{\prime}\right)} \mathcal{Q} i \mathbf{k} \cdot \mathbf{c} \mathcal{P} \delta \chi_{\mathbf{k}}\left(\mathbf{c}, s^{\prime}\right) .
$$

Inserting the above equation into the expression of the pressure tensor and taking into account that

$$
\int d \mathbf{c} \Delta_{j p}(\mathbf{c}) \mathcal{Q} \Lambda(\mathbf{c}) \mathcal{Q} h(\mathbf{c})=\int d \mathbf{c} \Delta_{j p}(\mathbf{c}) \Lambda(\mathbf{c}) h(\mathbf{c}),
$$

we obtain that

$$
\begin{aligned}
\Pi_{\mathbf{k}, j p}(s) \approx & -\int d \mathbf{c} \Delta_{j p}(\mathbf{c}) \int_{0}^{s} d s^{\prime} e^{\Lambda\left(s-s^{\prime}\right)} i \mathbf{k} \cdot \mathbf{c} \mathcal{P} \delta \chi_{\mathbf{k}}\left(\mathbf{c}, s^{\prime}\right) \\
= & -\int d \mathbf{c} \Delta_{j p}(\mathbf{c}) \int_{0}^{s} d s^{\prime} e^{\Lambda\left(s-s^{\prime}\right)} i \mathbf{k} \\
& \cdot \mathbf{c} \sum_{\beta=1}^{d+2}\left\langle\bar{\xi}_{\beta}(\mathbf{c}) \mid \delta \chi_{\mathbf{k}}\left(\mathbf{c}, s^{\prime}\right)\right\rangle \xi_{\beta}(\mathbf{c}) \\
= & -\sum_{r} \sum_{q} i k_{r} \int_{0}^{s} d s^{\prime} w_{\mathbf{k}, q}\left(s^{\prime}\right) \\
& \times \int d \mathbf{c} \Delta_{j p}(\mathbf{c}) e^{\Lambda\left(s-s^{\prime}\right)} c_{r} \xi_{2, q}(\mathbf{c}),
\end{aligned}
$$

where we have taken into account that there is no coupling with the density nor with the temperature. Finally, by symmetry considerations, we arrive at the expression used in the main text,

$$
\begin{aligned}
\Pi_{\mathbf{k}, j p}(s) \approx & -i \int_{0}^{s} d s^{\prime} G_{x y}\left(s-s^{\prime}\right)\left[k_{j} w_{\mathbf{k}, p}\left(s^{\prime}\right)+k_{p} w_{\mathbf{k}, j}\left(s^{\prime}\right)\right. \\
& \left.-\frac{2}{d} \delta_{j p} \mathbf{k} \cdot \mathbf{w}_{\mathbf{k}}\left(s^{\prime}\right)\right],
\end{aligned}
$$

where

$$
G_{x y}(s)=\int d \mathbf{c} \Delta_{x y}(\mathbf{c}) e^{\Lambda s} c_{x} \xi_{2, y}(\mathbf{c}) .
$$

To evaluate the heat flux to first order in $k$, we substitute expression (C2) into the heat flux and. taking into account

$$
\int d \mathbf{c} \Sigma_{j}(\mathbf{c}) \mathcal{Q} \Lambda(\mathbf{c}) \mathcal{Q} h(\mathbf{c})=\int d \mathbf{c} \Sigma_{j}(\mathbf{c}) \Lambda(\mathbf{c}) h(\mathbf{c}),
$$

we obtain

$$
\begin{aligned}
\phi_{\mathbf{k}, j}(s) \approx & -\int d \mathbf{c} \Sigma_{j}(\mathbf{c}) \int_{0}^{s} d s^{\prime} e^{\Lambda\left(s-s^{\prime}\right)} i \mathbf{k} \cdot \mathbf{c} \mathcal{P} \delta \chi_{\mathbf{k}}\left(\mathbf{c}, s^{\prime}\right) \\
= & -\int d \mathbf{c} \Sigma_{j}(\mathbf{c}) \int_{0}^{s} d s^{\prime} e^{\Lambda\left(s-s^{\prime}\right)} i \mathbf{k} \\
& \cdot \mathbf{c} \sum_{\beta=1}^{d+2}\left\langle\bar{\xi}_{\beta}(\mathbf{c}) \mid \delta \chi_{\mathbf{k}}\left(\mathbf{c}, s^{\prime}\right)\right\rangle \xi_{\beta}(\mathbf{c}) \\
= & -i k_{j} \int d \mathbf{c} \Sigma_{j}(\mathbf{c}) \int_{0}^{s} d s^{\prime} e^{\Lambda\left(s-s^{\prime}\right)} c_{j} \\
& \times\left[\left\langle\bar{\xi}_{1}(\mathbf{c}) \mid \delta \chi_{\mathbf{k}}\left(\mathbf{c}, s^{\prime}\right)\right\rangle \xi_{1}(c)+\left\langle\bar{\xi}_{3}(\mathbf{c}) \mid \delta \chi_{\mathbf{k}}\left(\mathbf{c}, s^{\prime}\right)\right\rangle \xi_{3}(c)\right],
\end{aligned}
$$

where we have used that there is no coupling with the flow velocity. Finally, taking into account Eqs. (62) and (64) and symmetry considerations, we arrive at the expression of the main text,

$$
\begin{aligned}
\phi_{\mathbf{k}, j}(s)= & -i k_{j} \int_{0}^{s} d s^{\prime}\left\{\rho_{\mathbf{k}}\left(s^{\prime}\right)\left[H_{1}\left(s-s^{\prime}\right)+\frac{1}{3} H_{3}\left(s-s^{\prime}\right)\right]\right. \\
& \left.+\frac{1}{2} \theta_{\mathbf{k}}\left(s^{\prime}\right) H_{3}\left(s-s^{\prime}\right)\right\},
\end{aligned}
$$

where

$$
H_{j}(s)=\int d \mathbf{c} \Sigma_{x}(\mathbf{c}) e^{\Lambda s} c_{x} \xi_{j}(\mathbf{c}), \quad j=1,3 .
$$

\section{APPENDIX D: EVALUATION OF THE COOLING RATE TO SECOND ORDER IN $k$}

We work out here the cooling rate given by Eq. (75) to second order in $k$. As in Appendix $\mathrm{C}$, choosing an initial condition with $\mathcal{Q} \delta \chi_{\mathbf{k}}(\mathbf{c}, 0)=0$, and neglecting the $k$ contribution in the kinetic modes, we have to $k^{2}$ order in the hydrodynamic fields

$$
\begin{aligned}
\mathcal{Q} \delta \chi_{\mathbf{k}}(\mathbf{c}, s) & \\
\approx & -\int_{0}^{s} d s^{\prime} e^{\mathcal{Q} \Lambda \mathcal{Q}\left(s-s^{\prime}\right)} \mathcal{Q} i \mathbf{k} \cdot \mathbf{c} \mathcal{P} \delta \chi_{\mathbf{k}}\left(\mathbf{c}, s^{\prime}\right) \\
& -\int_{0}^{s} d s^{\prime} \int_{0}^{s-s^{\prime}} d s^{\prime \prime} \mathcal{Q} e^{\Lambda\left(s-s^{\prime}-s^{\prime \prime}\right)} \mathbf{k} \cdot \mathbf{c} \mathcal{Q} e^{\Lambda s^{\prime \prime}} \mathbf{k} \cdot \mathbf{c} \mathcal{P} \delta \chi_{\mathbf{k}}\left(\mathbf{c}, s^{\prime}\right),
\end{aligned}
$$


where the identity for any two operators $A$ and $B$

$$
e^{(A+B) t}=e^{A t}+\int_{0}^{t} d t^{\prime} e^{A\left(t-t^{\prime}\right)} B e^{(A+B) t^{\prime}},
$$

has been used in order to perform the expansion. The first term of the right-hand side of (D1) is the first order in $k$ contribution, $\mathcal{Q} \delta \chi_{\mathbf{k}}(\mathbf{c}, s)^{(1)}$, calculated in Appendix C, while the second term is the $k^{2}$ contribution, $\mathcal{Q} \delta \chi_{\mathbf{k}}(\mathbf{c}, s)^{(2)}$. By substituting (D1) into (75) the cooling rate to $k^{2}$ order is obtained. The first order contribution is

$$
\begin{aligned}
\delta \zeta_{\mathbf{k}}^{(1)}(s) & =-\int d \mathbf{c} \frac{2 c^{2}}{d} \Lambda(\mathbf{c}) \int_{0}^{s} d s^{\prime} \mathcal{Q} e^{\Lambda\left(s-s^{\prime}\right)} i \mathbf{k} \cdot \mathbf{c} \mathcal{P} \delta \chi_{\mathbf{k}}\left(\mathbf{c}, s^{\prime}\right) \\
& =-2\left\langle\bar{\xi}_{3}(c) \mid \Lambda(\mathbf{c}) \int_{0}^{s} d s^{\prime} \mathcal{Q} e^{\Lambda\left(s-s^{\prime}\right)} i \mathbf{k} \cdot \mathbf{c} \mathcal{P} \delta \chi_{\mathbf{k}}\left(\mathbf{c}, s^{\prime}\right)\right\rangle
\end{aligned}
$$

where we have used Eq. (53). Taking into account that

$$
\left\langle\bar{\xi}_{3}(c) \mid \Lambda(\mathbf{c}) \mathcal{Q} h(\mathbf{c})\right\rangle=\left\langle\bar{\xi}_{3}(c) \mid\left[\Lambda(\mathbf{c})-\lambda_{3}\right] h(\mathbf{c})\right\rangle,
$$

it can be rewritten as

$$
\begin{aligned}
\delta \zeta_{\mathbf{k}}^{(1)}(s) & \\
= & -2\left\langle\bar{\xi}_{3}(c) \mid\left[\Lambda(\mathbf{c})-\lambda_{3}\right] \int_{0}^{s} d s^{\prime} e^{\Lambda\left(s-s^{\prime}\right)} i \mathbf{k} \cdot \mathbf{c} \mathcal{P} \delta \chi_{\mathbf{k}}\left(\mathbf{c}, s^{\prime}\right)\right\rangle \\
= & -2 i \sum_{p} k_{p} \int_{0}^{s} d s^{\prime} w_{\mathbf{k}, p}\left(s^{\prime}\right)\left\langle\bar{\xi}_{3}(c)\right| \\
& \left.\times\left[\Lambda(\mathbf{c})-\lambda_{3}\right] e^{\Lambda\left(s-s^{\prime}\right)} c_{p} \xi_{2, p}(\mathbf{c})\right\rangle,
\end{aligned}
$$

where we have used that, by symmetry considerations, there is only coupling with the flow velocity. Finally, we can transform (D5) to write it as in the main text

$$
\delta \zeta_{\mathbf{k}}(s)^{(1)}=-2 i \int_{0}^{s} d s^{\prime} \mathbf{k} \cdot \mathbf{w}_{\mathbf{k}}\left(s^{\prime}\right) Z\left(s-s^{\prime}\right),
$$

where

$$
Z(s)=\left\langle\bar{\xi}_{3}(\mathbf{c}) \mid\left[\Lambda(\mathbf{c})-\lambda_{3}\right] e^{\Lambda s} c_{x} \xi_{2, x}(\mathbf{c})\right\rangle .
$$

By similar manipulations, the second order in $k$ contribution can be written as

$$
\begin{aligned}
\delta \zeta_{\mathbf{k}}^{(2)}(s) & =-2\left\langle\bar{\xi}_{3}(c) \mid\left[\Lambda(\mathbf{c})-\lambda_{3}\right] \int_{0}^{s} d s^{\prime} \int_{0}^{s-s^{\prime}} d s^{\prime \prime} e^{\Lambda\left(s-s^{\prime}-s^{\prime \prime}\right)} \mathbf{k} \cdot \mathbf{c} \mathcal{Q} e^{\Lambda s^{\prime \prime}} \mathbf{k} \cdot \mathbf{c} \mathcal{P} \delta \chi_{\mathbf{k}}\left(\mathbf{c}, s^{\prime}\right)\right\rangle \\
& =-2\left\langle\bar{\xi}_{3}(c) \mid\left[\Lambda(\mathbf{c})-\lambda_{3}\right] \int_{0}^{s} d s^{\prime} \int_{0}^{s-s^{\prime}} d s^{\prime \prime} e^{\Lambda\left(s-s^{\prime}-s^{\prime \prime}\right)} \mathbf{k} \cdot \mathbf{c} \mathcal{Q} e^{\Lambda s^{\prime \prime}} \mathbf{k} \cdot \mathbf{c}\left[\rho_{\mathbf{k}}\left(s^{\prime}\right) \xi_{1}(c)+\left\langle\bar{\xi}_{3}(c) \mid \delta \chi_{\mathbf{k}}\left(\mathbf{c}, s^{\prime}\right)\right\rangle \xi_{3}(c)\right]\right\rangle \\
& =-2 k^{2}\left\langle\bar{\xi}_{3}(c) \mid\left[\Lambda(\mathbf{c})-\lambda_{3}\right] \int_{0}^{s} d s^{\prime} \int_{0}^{s-s^{\prime}} d s^{\prime \prime} e^{\Lambda\left(s-s^{\prime}-s^{\prime \prime}\right)} c_{x} \mathcal{Q} e^{\Lambda s^{\prime \prime}} c_{x}\left[\rho_{\mathbf{k}}\left(s^{\prime}\right) \xi_{1}(c)+\left\langle\bar{\xi}_{3}(c) \mid \delta \chi_{\mathbf{k}}\left(\mathbf{c}, s^{\prime}\right)\right\rangle \xi_{3}(c)\right]\right\rangle
\end{aligned}
$$

which can be recast as

$$
\begin{aligned}
\delta \zeta_{\mathbf{k}}^{(2)}(s)= & -2 k^{2} \int_{0}^{s} d s^{\prime}\left[\rho_{\mathbf{k}}\left(s^{\prime}\right) Z_{1}\left(s-s^{\prime}\right)\right. \\
& \left.+\left\langle\bar{\xi}_{3}(c) \mid \delta \chi_{\mathbf{k}}\left(\mathbf{c}, s^{\prime}\right)\right\rangle Z_{3}\left(s-s^{\prime}\right)\right],
\end{aligned}
$$

where

$$
\begin{aligned}
Z_{j}(s) & =\left\langle\bar{\xi}_{3}(\mathbf{c}) \mid\left[\Lambda(\mathbf{c})-\lambda_{3}\right] \int_{0}^{s} d s^{\prime} e^{\Lambda\left(s-s^{\prime}\right)} c_{x} \mathcal{Q} e^{\Lambda s^{\prime}} c_{x} \xi_{j}(\mathbf{c})\right\rangle \\
j & =1,3
\end{aligned}
$$

or, as written in the main text,

$$
\begin{aligned}
\delta \zeta_{\mathbf{k}}^{(2)}(s)= & -2 k^{2} \int_{0}^{s} d s^{\prime}\left\{\rho_{\mathbf{k}}\left(s^{\prime}\right)\left[Z_{1}\left(s-s^{\prime}\right)+\frac{1}{3} Z_{3}\left(s-s^{\prime}\right)\right]\right. \\
& \left.+\frac{1}{2} \theta_{\mathbf{k}}\left(s^{\prime}\right) Z_{3}\left(s-s^{\prime}\right)\right\}
\end{aligned}
$$

\section{APPENDIX E: ANALYSIS OF THE EQUATION FOR THE TRANSVERSAL VELOCITY}

In Laplace space, the transversal velocity is given by Eq. (92),

$$
\bar{w}_{\mathbf{k}, \perp}(z)=\frac{w_{\mathbf{k}, \perp}(0)}{z+k^{2} \bar{G}_{x y}(z)},
$$

where we have skipped the superscript $j$. This formula depends on $\bar{G}_{x y}(z)$ that, in principle, can be a complicated object. We already know that in real time it decays with the kinetic modes and in the free-cooling case it has been shown numerically that it is very well fitted by a single exponential (single mode approximation). Let us consider this simple form for the general case can be performed in a similar way. In this approximation, we have

$$
G_{x y}(s)=C e^{-\lambda s}, \quad \bar{G}_{x y}(z)=\frac{C}{z+\lambda},
$$

and Eq. (E1) is just

$$
\bar{w}_{\mathbf{k}, \perp}(z)=\frac{w_{\mathbf{k}, \perp}(0)(z+\lambda)}{z(z+\lambda)+C k^{2}},
$$


which can be inverted exactly in terms of two exponentials [49]. The function given by Eq. (E3) has the two poles

$$
\begin{gathered}
z_{1}(k)=\frac{-\lambda+\sqrt{\lambda^{2}-4 C k^{2}}}{2} \approx-\frac{C}{\lambda} k^{2}, \\
z_{2}(k)=\frac{-\lambda-\sqrt{\lambda^{2}-4 C k^{2}}}{2} \approx-\lambda+\frac{C}{\lambda} k^{2},
\end{gathered}
$$

and it can be written as

$$
\bar{w}_{\mathbf{k}, \perp}(z)=\frac{A(k)}{z-z_{1}(k)}+\frac{B(k)}{z-z_{2}(k)} .
$$

We then have

$$
\begin{aligned}
& w_{\mathbf{k}, \perp}(0)(z+\lambda) \\
& \quad=A(k)\left[z-z_{2}(k)\right]+B(k)\left[z-z_{1}(k)\right] \\
& \quad \approx A(k)\left(z+\lambda-\frac{C}{\lambda} k^{2}\right)+B(k)\left(z+\frac{C}{\lambda} k^{2}\right),
\end{aligned}
$$

with which we identify the constant $A$ and $B$ to zeroth order in $k$,

$$
A \approx w_{\mathbf{k}, \perp}(0), \quad B \approx 0 .
$$

With Eqs. (E6) and (E8) we obtain the expression for the transversal velocity of the main text, Eq. (96),

$$
w_{\mathbf{k}, \perp}(s) \approx w_{\mathbf{k}, \perp}(0) e^{-\eta k^{2} s},
$$

with

$$
\eta=\frac{C}{\lambda}=\int_{0}^{\infty} d s G_{x y}(s) .
$$

If the function $G_{x y}(s)$ is a linear combination of kinetic modes, the analysis can be performed following the same lines obtaining Eq. (E9) with $\eta=\int_{0}^{\infty} d s G_{x y}(s)$.

\section{APPENDIX F: ANALYSIS OF THE COUPLED HYDRODYNAMIC EQUATIONS}

We evaluate here the asymptotic behavior of Eq. (93),

$$
[z I+A(k, z)]\left(\begin{array}{c}
\bar{\rho}_{\mathbf{k}}(z) \\
\bar{w}_{\mathbf{k}, \|}(z) \\
\bar{\theta}_{\mathbf{k}}(z)
\end{array}\right)=\left(\begin{array}{c}
\rho_{\mathbf{k}}(0) \\
w_{\mathbf{k}, \|}(0) \\
\theta_{\mathbf{k}}(0)
\end{array}\right)
$$

in the hydrodynamic limit. The matrix $A(k, z)$ is given in Eq. (94) and can be written as

$$
A(k, z)=A_{0}+i k A_{1}(z)+k^{2} A_{2}(z),
$$

where

$$
\begin{array}{cc}
A_{0}=\left(\begin{array}{ccc}
0 & 0 & 0 \\
0 & 0 & 0 \\
\frac{2}{3} \gamma & 0 & \gamma
\end{array}\right), & A_{1}(z)=\left(\begin{array}{ccc}
0 & 1 & 0 \\
\frac{1}{2} & 0 & \frac{1}{2} \\
0 & \bar{q}(z) & 0
\end{array}\right), \\
A_{2}(z)=\left(\begin{array}{ccc}
0 & 0 & 0 \\
0 & 2 \frac{d-1}{d} \bar{G}_{x y}(z) & 0 \\
\frac{2}{d} \bar{G}_{1}(z) & 0 & \frac{2}{d} \bar{G}_{3}(z)
\end{array}\right) .
\end{array}
$$

In terms of the eigenvalues and eigenfunctions of the matrix $A(k, z)$

$$
A(k, z)\left|\psi_{\beta}(k, z)\right\rangle=a_{\beta}(k, z)\left|\psi_{\beta}(k, z)\right\rangle,
$$

the solution can be written explicitly as

$$
\begin{aligned}
|\bar{y}(\mathbf{k}, z)\rangle & =[z I+A(k, z)]^{-1}|y(\mathbf{k}, 0)\rangle \\
& =\sum_{\beta=1}^{3} \frac{\left\langle\bar{\psi}_{\beta}(k, z) \mid y(\mathbf{k}, 0)\right\rangle}{z+a_{\beta}(k, z)}\left|\psi_{\beta}(k, z)\right\rangle,
\end{aligned}
$$

where we have introduced the notation

$$
|y(\mathbf{k}, s)\rangle \equiv\left(\begin{array}{c}
\rho_{\mathbf{k}}(s) \\
w_{\mathbf{k}, \|}(s) \\
\theta_{\mathbf{k}}(s)
\end{array}\right), \quad|\bar{y}(\mathbf{k}, z)\rangle \equiv\left(\begin{array}{c}
\bar{\rho}_{\mathbf{k}}(z) \\
\bar{w}_{\mathbf{k}, \|}(z) \\
\bar{\theta}_{\mathbf{k}}(z)
\end{array}\right),
$$

and the functions $\left\{\left\langle\bar{\psi}_{\beta}(k, z)\right|\right\}_{\beta=1}^{3}$ are the left eigenfunctions of $A(k, z)$. Let us introduce the expansion in powers of $k$ of the eigenvalues and eigenfunctions

$$
\begin{array}{r}
\left|\psi_{\beta}(k, z)\right\rangle=\left|\psi_{\beta}^{(0)}(z)\right\rangle+k\left|\psi_{\beta}^{(1)}(z)\right\rangle+k^{2}\left|\psi_{\beta}^{(2)}(z)\right\rangle+\cdots, \\
a_{\beta}(k, z)=a_{\beta}^{(0)}(z)+k a_{\beta}^{(1)}(z)+k^{2} a_{\beta}^{(2)}(z)+\cdots
\end{array}
$$

In the hydrodynamic limit, and applying the same ideas as in Appendix E, we obtain

$$
|\bar{y}(\mathbf{k}, z)\rangle \approx \sum_{\beta} \frac{\left\langle\phi_{\beta} \mid y(\mathbf{k}, 0)\right\rangle}{z-\lambda_{\beta}(k)}\left|\psi_{\beta}^{(0)}\right\rangle,
$$

where $\left\{\lambda_{\beta}(k)\right\}_{\beta=1}^{3}$ are the hydrodynamic eigenvalues that appear as the smallest root of equation $z+a_{\beta}(k, z)=0$. The set $\left\{\left\langle\phi_{\beta}\right|\right\}_{\beta=1}^{3}$ is the biorthogonal set constructed to have $\left\langle\phi_{\beta} \mid \psi_{\beta^{\prime}}^{(0)}\right\rangle=\delta_{\beta, \beta^{\prime}}$. The problem is then to calculate the sets $\left\{\left|\psi_{\beta}^{(0)}\right\rangle\right\}_{\beta=1}^{3}, \quad\left\{\left\langle\phi_{\beta}\right|\right\}_{\beta=1}^{3}$ and $\left\{a_{\beta}(k, z)\right\}_{\beta=1}^{3}$. As $\left\{\lambda_{\beta}(k)\right\}_{\beta=1}^{3}$ is needed to $k^{2}$ order, $\left\{a_{\beta}(k, z)\right\}_{\beta=1}^{3}$ have to be calculated at the same order, which can be done by use of standard perturbation theory.

The eigenvalues of $A_{0}$ can be easily calculated, obtaining

$$
a_{1}^{(0)}=a_{2}^{(0)}=0, \quad a_{3}^{(0)}=\gamma,
$$

so the vanishing eigenvalue is twofold degenerate. The corresponding eigenfunctions are

$$
\left|u_{1}\right\rangle=\left(\begin{array}{c}
3 \\
0 \\
-2
\end{array}\right), \quad\left|u_{2}\right\rangle=\left(\begin{array}{c}
3 \\
1 \\
-2
\end{array}\right), \quad\left|u_{3}\right\rangle=\left|\psi_{3}^{(0)}\right\rangle=\left(\begin{array}{l}
0 \\
0 \\
1
\end{array}\right)
$$

and the biorthogonal set is

$\left\langle v_{1}\right|=\left(\frac{1}{3},-1,0\right), \quad\left\langle v_{2}\right|=(0,1,0), \quad\left\langle v_{3}\right|=\left\langle\phi_{3}\right|=\left(\frac{2}{3}, 0,1\right)$.

In the nondegenerate case, the first contributions to the expansion are

$$
a_{3}^{(1)}(z)=\left\langle v_{3}\left|i A_{1}\right| u_{3}\right\rangle=0
$$

and

$$
\begin{aligned}
a_{3}^{(2)}(z) & =\left\langle v_{3}\left|A_{2}\right| u_{3}\right\rangle+\frac{1}{\gamma} \sum_{n \neq 3}\left\langle v_{n}\left|i A_{1}\right| u_{3}\right\rangle\left\langle v_{3}\left|i A_{1}\right| u_{n}\right\rangle \\
& =\frac{2}{d} \bar{G}_{3}(z)-\frac{1}{3 \gamma}-\frac{q(z)}{2 \gamma},
\end{aligned}
$$


so we have

$$
a_{3}(k, z) \approx \gamma+\left[\frac{2}{d} \bar{G}_{3}(z)-\frac{1}{3 \gamma}-\frac{q(z)}{2 \gamma}\right] k^{2} .
$$

For the degenerate case, we first have to consider the submatrix $i A_{1}^{(S)}(z)=\left(\begin{array}{ll}\left\langle v_{1}\left|i A_{1}\right| u_{1}\right\rangle & \left\langle v_{1}\left|i A_{1}\right| u_{2}\right\rangle \\ \left\langle v_{2}\left|i A_{1}\right| u_{1}\right\rangle & \left\langle v_{2}\left|i A_{1}\right| u_{2}\right\rangle\end{array}\right)=i\left(\begin{array}{cc}-\frac{1}{2} & -\frac{1}{6} \\ \frac{1}{2} & \frac{1}{2}\end{array}\right)$.

Its eigenvalues are the first-order correction of the degenerate eigenvalues

$$
a_{1}^{(1)}(z)=-\frac{i}{\sqrt{6}}, \quad a_{2}^{(1)}(z)=\frac{i}{\sqrt{6}},
$$

and its eigenfunctions give us the components of the corresponding zeroth-order eigenfunction,

$$
\begin{aligned}
& \left(\begin{array}{l}
\left\langle v_{1} \mid \psi_{1}^{(0)}\right\rangle \\
\left\langle v_{2} \mid \psi_{1}^{(0)}\right\rangle
\end{array}\right)=\left(\begin{array}{c}
-1-\sqrt{\frac{2}{3}} \\
1
\end{array}\right), \\
& \left(\begin{array}{c}
\left\langle v_{1} \mid \psi_{2}^{(0)}\right\rangle \\
\left\langle v_{2} \mid \psi_{2}^{(0)}\right\rangle
\end{array}\right)=\left(\begin{array}{c}
-1+\sqrt{\frac{2}{3}} \\
1
\end{array}\right),
\end{aligned}
$$

SO

$$
\left|\psi_{1}^{(0)}\right\rangle=\left(\begin{array}{c}
-6 \\
\sqrt{6} \\
4
\end{array}\right), \quad\left|\psi_{2}^{(0)}\right\rangle=\left(\begin{array}{c}
6 \\
\sqrt{6} \\
-4
\end{array}\right)
$$

which do not depend on $z$. The corresponding biorthogonal functions can also be calculated, obtaining

$$
\left\langle\phi_{1}\right|=\left(-\frac{1}{12}, \frac{1}{2 \sqrt{6}}, 0\right),\left\langle\phi_{2}\right|=\left(\frac{1}{12}, \frac{1}{2 \sqrt{6}}, 0\right) .
$$

With these functions, the $k^{2}$ corrections to the degenerate eigenvalues follow from straightforward calculations,

$$
\begin{aligned}
a_{1}^{(2)}(z) & =\left\langle\phi_{1}\left|A_{2}\right| \psi_{1}^{(0)}\right\rangle-\frac{1}{\gamma}\left\langle\phi_{3}\left|i A_{1}\right| \psi_{1}^{(0)}\right\rangle\left\langle\phi_{1}\left|i A_{1}\right| \psi_{3}^{(0)}\right\rangle \\
& =\frac{d-1}{d} \bar{G}_{x y}(z)+\frac{1}{4 \gamma}\left[\frac{2}{3}+q(z)\right]
\end{aligned}
$$

and

$$
\begin{aligned}
a_{2}^{(2)}(z) & =\left\langle\phi_{2}\left|A_{2}\right| \psi_{2}^{(0)}\right\rangle-\frac{1}{\gamma}\left\langle\phi_{3}\left|i A_{1}\right| \psi_{2}^{(0)}\right\rangle\left\langle\phi_{2}\left|i A_{1}\right| \psi_{3}^{(0)}\right\rangle \\
& =\frac{d-1}{d} \bar{G}_{x y}(z)+\frac{1}{4 \gamma}\left[\frac{2}{3}+q(z)\right],
\end{aligned}
$$

so we have

$$
\begin{aligned}
& a_{1}(k, z) \approx-\frac{i}{\sqrt{6}} k+\left[\frac{d-1}{d} \bar{G}_{x y}(z)+\frac{1}{6 \gamma}+\frac{q(z)}{4 \gamma}\right] k^{2}, \\
& a_{2}(k, z) \approx+\frac{i}{\sqrt{6}} k+\left[\frac{d-1}{d} \bar{G}_{x y}(z)+\frac{1}{6 \gamma}+\frac{q(z)}{4 \gamma}\right] k^{2} .
\end{aligned}
$$

The smallest root of $z+a_{\beta}(k, z)=0$, with $a_{\beta}(k, z)$ given by Eqs. (F24), (F25), and (F16), are the hydrodynamic eigenvalues given in the main text, i.e., Eqs. (103)-(105).

\section{APPENDIX G: EVALUATION OF \\ THE KINETIC EIGENVALUES}

Let us assume

$$
\Lambda^{+}(\mathbf{c}) \chi_{s}(c) \Delta_{j p}(\mathbf{c}) \approx \lambda_{N H}^{(1)} \chi_{s}(c) \Delta_{j p}(\mathbf{c})
$$

Multiplying by $c_{x} c_{y}$ and integrating we obtain

$$
\begin{aligned}
\lambda_{N H}^{(1)} \int d \mathbf{c} c_{x}^{2} c_{y}^{2} \chi_{s}(c) & =\int d \mathbf{c} c_{x} c_{y} \Lambda^{+}(\mathbf{c}) \chi_{s}(c) c_{x} c_{y} \\
& =\int d \mathbf{c} c_{x} c_{y} \Lambda(\mathbf{c}) \chi_{s}(c) c_{x} c_{y},
\end{aligned}
$$

so we have

$$
\lambda_{N H}^{(1)}=\frac{4}{1+a_{2}^{s}} I_{1}, \quad I_{1}=\int d \mathbf{c} c_{x} c_{y} \Lambda(\mathbf{c}) \chi_{s}(c) c_{x} c_{y} .
$$

The heating does not contribute to the integral $I_{1}$ and we have

$$
I_{1}=\int d \mathbf{c}_{1} \int d \mathbf{c}_{2} c_{1 x} c_{1 y} \bar{T}_{0}\left(\mathbf{c}_{1}, \mathbf{c}_{2}\right)\left(1+P_{12}\right) \chi_{s}\left(c_{1}\right) \chi_{s}\left(c_{2}\right) c_{1 x} c_{1 y} .
$$

Taking into account

$$
\begin{aligned}
\left(b_{\sigma}-1\right)\left(c_{1 x} c_{1 y}+c_{2 x} c_{2 y}\right) & \\
= & \frac{(1+\alpha)^{2}}{2}\left(\hat{\boldsymbol{\sigma}} \cdot \mathbf{c}_{12}\right)^{2} \hat{\sigma}_{x} \hat{\sigma}_{y} \\
& \quad-\frac{1+\alpha}{2}\left(\hat{\boldsymbol{\sigma}} \cdot \mathbf{c}_{12}\right)\left(c_{12 y} \hat{\sigma}_{x}+c_{12 x} \hat{\sigma}_{y}\right),
\end{aligned}
$$

and the solid angle integrals

$$
\begin{array}{r}
\int d \hat{\boldsymbol{\sigma}} \Theta\left(\hat{\boldsymbol{\sigma}} \cdot \mathbf{c}_{12}\right)\left(\hat{\boldsymbol{\sigma}} \cdot \mathbf{c}_{12}\right)^{3} \hat{\sigma}_{x} \hat{\sigma}_{y}=\frac{3 \pi^{\frac{d-1}{2}}}{2 \Gamma\left(\frac{d+5}{2}\right)} c_{12} c_{12 x} c_{12 y}, \\
\int d \hat{\boldsymbol{\sigma}} \Theta\left(\hat{\boldsymbol{\sigma}} \cdot \mathbf{c}_{12}\right)\left(\hat{\boldsymbol{\sigma}} \cdot \mathbf{c}_{12}\right)^{2} \hat{\sigma}_{j}=\frac{\pi^{\frac{d-1}{2}}}{\Gamma\left(\frac{d+3}{2}\right)} c_{12} c_{12 j},
\end{array}
$$

we finally obtain

$$
\begin{aligned}
I_{1}= & {\left[\frac{3(1+\alpha)^{2}}{4 \Gamma\left(\frac{d+5}{2}\right)}-\frac{1+\alpha}{\Gamma\left(\frac{d+3}{2}\right)}\right] \pi^{\frac{d-1}{2}} \int d \mathbf{c}_{1} } \\
& \times \int d \mathbf{c}_{2} \chi_{s}\left(c_{1}\right) \chi_{s}\left(c_{2}\right) c_{1 x} c_{1 y} c_{12} c_{12 x} c_{12 y} \\
= & -\frac{(2 d+3-3 \alpha)(1+\alpha) \pi^{\frac{d-1}{2}}}{2 \sqrt{2} d(d+2) \Gamma(d / 2)}\left(1+\frac{23}{16} a_{2}^{s}\right),
\end{aligned}
$$

where the last integral has been performed with $\chi_{s}(c)$ in the first Sonine approximation.

To calculate $\lambda_{\mathrm{NH}}^{(2)}$, we multiply

$$
\Lambda^{+}(\mathbf{c}) \chi_{s}(c) \Sigma_{j}(\mathbf{c}) \approx \lambda_{\mathrm{NH}}^{(2)} \chi_{s}(c) \Sigma_{j}(\mathbf{c})
$$

by $c_{x}$ and proceed with an integration over $\mathbf{c}$ to obtain

$$
\lambda_{\mathrm{NH}}^{(2)}=\frac{4}{(d+2) a_{2}^{s}} I_{2}, \quad I_{2}=\int d \mathbf{c} c_{x} c^{2} \Lambda(\mathbf{c}) \chi_{s}(c) c_{x} .
$$

The heating contribution to $I_{2}$ is simply

$$
I_{2}^{(H)}=\int d \mathbf{c} c_{x} c^{2} \frac{\tilde{\xi}^{2}}{2} \frac{\partial^{2}}{\partial c^{2}} \chi_{s}(c) c_{x}=\frac{d+2}{2} \widetilde{\xi}^{2},
$$


where $\tilde{\xi}^{2}$ is given in Eq. (38) and the collisional term is

$$
I_{2}^{(C)}=\int d \mathbf{c}_{1} c_{1 x} c_{1}^{2} \int d \mathbf{c}_{2} \bar{T}_{0}\left(\mathbf{c}_{1}, \mathbf{c}_{2}\right)\left(1+P_{12}\right) \chi_{s}\left(c_{1}\right) \chi_{s}\left(c_{2}\right) c_{1 x} .
$$

Taking into account

$$
\begin{aligned}
\left(b_{\sigma}\right. & -1)\left(c_{1}^{2} c_{1 x}+c_{2}^{2} c_{2 x}\right) \\
= & \left(\frac{1+\alpha}{2}\right)^{2}\left(\hat{\boldsymbol{\sigma}} \cdot \mathbf{c}_{12}\right)^{2}\left[c_{1 x}+c_{2 x}+2\left(\hat{\boldsymbol{\sigma}} \cdot \mathbf{c}_{1}\right) \hat{\sigma}_{x}\right. \\
& \left.+2\left(\hat{\boldsymbol{\sigma}} \cdot \mathbf{c}_{2}\right) \hat{\sigma}_{x}\right]-\frac{1+\alpha}{2}\left(\hat{\boldsymbol{\sigma}} \cdot \mathbf{c}_{12}\right)\left[c_{1}^{2} \hat{\sigma}_{x}-c_{2}^{2} \hat{\sigma}_{x}\right. \\
& \left.+2\left(\hat{\boldsymbol{\sigma}} \cdot \mathbf{c}_{1}\right) c_{1 x}-2\left(\hat{\boldsymbol{\sigma}} \cdot \mathbf{c}_{2}\right) c_{2 x}\right]
\end{aligned}
$$

and the solid angle integrals

$$
\begin{gathered}
\int d \hat{\boldsymbol{\sigma}} \Theta\left(\hat{\boldsymbol{\sigma}} \cdot \mathbf{c}_{12}\right)\left(\hat{\boldsymbol{\sigma}} \cdot \mathbf{c}_{12}\right)^{3}=\frac{\pi^{\frac{d-1}{2}}}{\Gamma\left(\frac{d+3}{2}\right)} c_{12}^{3}, \\
\int d \hat{\boldsymbol{\sigma}} \Theta\left(\hat{\boldsymbol{\sigma}} \cdot \mathbf{c}_{12}\right)\left(\hat{\boldsymbol{\sigma}} \cdot \mathbf{c}_{12}\right)^{3}\left(\hat{\boldsymbol{\sigma}} \cdot \mathbf{c}_{1}\right) \hat{\sigma}_{x} \\
=\frac{\pi^{\frac{d-1}{2}}}{2 \Gamma\left(\frac{d+5}{2}\right)}\left[c_{12}^{3} c_{1 x}+3 c_{12} c_{12 x}\left(\mathbf{c}_{1} \cdot \mathbf{c}_{12}\right)\right],
\end{gathered}
$$

(G15) we have

$$
I_{2}^{(C)}=\frac{(1+\alpha) \pi^{\frac{d-1}{2}}}{2 \Gamma\left(\frac{d+3}{2}\right)} \int d \mathbf{c}_{1} \int d \mathbf{c}_{2} \chi_{s}\left(c_{1}\right) \chi_{s}\left(c_{2}\right) c_{1 x} \mathcal{F}\left(\mathbf{c}_{1}, \mathbf{c}_{2}\right),
$$

where

$$
\begin{aligned}
\mathcal{F}\left(\mathbf{c}_{1}, \mathbf{c}_{2}\right)= & \frac{1+\alpha}{d+3}\left[c_{12}^{3}\left(c_{1 x}+c_{2 x}\right)+3 c_{12} c_{12 x}\left(\mathbf{c}_{1}+\mathbf{c}_{2}\right) \cdot \mathbf{c}_{12}\right] \\
& -\left(c_{1}^{2}-c_{2}^{2}\right) c_{12} c_{12 x}+\frac{1+\alpha}{2} c_{12}^{3}\left(c_{1 x}+c_{2 x}\right) \\
& +2 c_{12}\left[\left(\mathbf{c}_{12} \cdot \mathbf{c}_{2}\right) c_{2 x}-\left(\mathbf{c}_{12} \cdot \mathbf{c}_{1}\right) c_{1 x}\right] .
\end{aligned}
$$

Evaluating the integral in the first Sonine approximation we get

$$
\begin{aligned}
I_{2}^{(C)}= & -\frac{(1+\alpha) \pi^{\frac{d-1}{2}}}{32 \sqrt{2} d \Gamma(d / 2)}\{(32+16 d)(1-\alpha) \\
& \left.+a_{2}^{s}[70+47 d-3(34+5 d) \alpha]\right\} .
\end{aligned}
$$

The eigenvalue can be finally written as

$$
\lambda_{\mathrm{NH}}^{(2)}=\frac{4}{(d+2) a_{2}^{s}}\left(\frac{d+2}{2} \widetilde{\xi}^{2}+I_{2}^{(C)}\right) .
$$

Let us note that the numerator goes as $a_{2}^{s}$ in the elastic limit, so a finite result is obtained in that limit.
[1] C. S. Campbell, Annu. Rev. Fluid Mech. 22, 57 (1990).

[2] P. K. Haff, J. Fluid. Mech. 134, 401 (1983).

[3] J. F. Lutsko, Phys. Rev. E 72, 021306 (2005).

[4] A. Goldshtein and M. Shapiro, J. Fluid. Mech. 282, 75 (1995).

[5] J. J. Brey, J. W. Dufty, and A. Santos, J. Stat. Phys. 87, 1051 (1997).

[6] P. Résibois and M. de Leener, Classical Kinetic Theory of Fluids (John Wiley, New York, 1977).

[7] J. J. Brey, J. W. Dufty, C. S. Kim, and A. Santos, Phys. Rev. E 58, 4638 (1998).

[8] J. W. Dufty and J. J. Brey, Phys. Rev. E 68, 030302(R) (2003).

[9] J. J. Brey, J. W. Dufty, and M. J. Ruiz-Montero, in Granular Gas Dynamics, edited by T. Poeschel and N. Brilliantov (Springer, Berlin, 2003).

[10] J. J. Brey and J. W. Dufty, Phys. Rev. E 72, 011303 (2005).

[11] J. W. Dufty and J. J. Brey, J. Stat. Phys. 109, 433 (2002).

[12] A. Baskaran, J. W. Dufty, and J. J. Brey, J. Stat. Mech. (2007) P12002.

[13] J. W. Dufty, A. Baskaran, and J. J. Brey, Phys. Rev. E 77, 031310 (2008).

[14] A. Baskaran, J. W. Dufty, and J. J. Brey, Phys. Rev. E 77, 031311 (2008).

[15] J. F. Lutsko, Phys. Rev. E 73, 021302 (2006).

[16] V. Garzó, Phys. Rev. E 73, 021304 (2006).

[17] J. M. Montanero and V. Garzó, Phys. Rev. E 67, 021308 (2003).

[18] V. Garzó and J. M. Montanero, Phys. Rev. E 68, 041302 (2003).

[19] A. Prevost, D. A. Egolf, and J. S. Urbach, Phys. Rev. Lett. 89, 084301 (2002).

[20] A. E. Lobkovsky, F. V. Reyes, and J. S. Urbach, Eur. Phys. J. Spec. Top. 179, 123 (2009).
[21] A. Puglisi, A. Gnoli, G. Gradenigo, A. Sarracino, and D. Villamaina, J. Chem. Phys. 136, 014704 (2012).

[22] V. Garzó, S. Tenneti, S. Subramaniam, and C. M. Hrenya, J. Fluid Mech. 712, 129 (2012).

[23] R. Cafiero, S. Luding, and H. J. Herrmann, Phys. Rev. Lett. 84, 6014 (2000).

[24] D. R. M. Williams and F. C. MacKintosh, Phys. Rev. E 54, R9 (1996).

[25] T. P. C. van Noije and M. H. Ernst, Granul. Matter 1, 57 (1998).

[26] T. P. C. van Noije, M. H. Ernst, E. Trizac, and I. Pagonabarraga, Phys. Rev. E 59, 4326 (1999).

[27] I. Pagonabarraga, E. Trizac, T. P. C. van Noije, and M. H. Ernst, Phys. Rev. E 65, 011303 (2001).

[28] J. M. Montanero and A. Santos, Granul. Matter 2, 53 (2000).

[29] V. Garzó and J. M. Montanero, Physica A 313, 336 (2002).

[30] M. H. Ernst, E. Trizac, and A. Barrat, Europhys. Lett. 76, 56 (2006).

[31] P. Visco, A. Puglisi, A. Barrat, E. Trizac, and F. van Wijland, Europhys. Lett. 72, 55 (2005); J. Stat. Phys. 125, 533 (2006).

[32] P. Visco, A. Puglisi, A. Barrat, F. van Wijland, and E. Trizac, Eur. Phys. J. B 51, 377 (2006).

[33] M. I. García de Soria, P. Maynar, and E. Trizac, Molec. Phys. 107, 383 (2009).

[34] P. Maynar, M. I. García de Soria, and E. Trizac, Eur. Phys. J. Spec. Top. 179, 123 (2009).

[35] K. Vollmayr-Lee, T. Aspelmeier, and A. Zippelius, Phys. Rev. E 83, 011301 (2011).

[36] For a variant of the model, see A. Puglisi, V. Loreto, U. Marini Betollo Marconi, and A. Vulpiani, Phys. Rev. E 59, 5582 (1999).

[37] V. Garzó, M. G. Chamorro, and F. V. Reyes, arXiv:1211.4985 [cond-mat.stat-mech]. 
[38] M. I. García de Soria, P. Maynar, and E. Trizac, Phys. Rev. E 85, 051301 (2012).

[39] N. G. van Kampen, Stochastic Processes in Physics and Chemistry (North-Holland, Amsterdam, 1992).

[40] A. Astillero and A. Santos, EPL 78, 24002 (2007).

[41] A. Astillero and A. Santos, Phys. Rev. E 85, 021302 (2012).

[42] A. F. Nikiforov and V. B. Uvarov, Special Functions of Mathematical Physics (Birkhäuser Verlag, Basel, 1988).

[43] P. Maynar and M. I. García de Soria, Math. Model. Nat. Phenom. 6, 87 (2011).
[44] J. A. McLennan, Introduction to Nonequilibrium Statistical Mechanics (Prentice-Hall, New Jersey, 1989).

[45] V. Garzó and J. W. Dufty, Phys. Rev. E 59, 5895 (1999).

[46] J. J. Brey, P. Maynar, and M. I. García de Soria, Phys. Rev. E 86, 061308 (2012)

[47] J. J. Brey, P. Maynar, and M. I. García de Soria, Phys. Rev. E 83, 041303 (2011).

[48] V. Garzó, Phys. Rev. E 84, 012301 (2011).

[49] J. P. Hansen and I. R. McDonald, Theory of Simple Liquids (Academic Press, Amsterdam, 2006). 